\title{
Inorganic Nanotubes with Permanent Wall Polarization as Dual Photo-Reactors for Wastewater Treatment with Simultaneous Fuel Production
}

\author{
Sabyasachi Patra, ${ }^{\mathrm{a}, \mathrm{d}, *}$ Delphine Schaming, ${ }^{\mathrm{b}}$ Pierre Picot, ${ }^{\mathrm{a}}$ Marie-Claire Pignié, ${ }^{\mathrm{a}}$ Jean-Blaise \\ Brubach, ${ }^{\mathrm{c}}$ Lorette Sicard, ${ }^{\mathrm{b}}$ Sophie Le Caër ${ }^{\mathrm{a}, *}$ Antoine Thill ${ }^{\mathrm{a}, *}$ \\ ${ }^{a}$ Université Paris-Saclay, CEA, CNRS, NIMBE, 91191, Gif-sur-Yvette, France \\ ${ }^{b}$ Université de Paris, ITODYS, CNRS, UMR 7086, 15 rue J.-A. de Baïf, F-75013 Paris, France \\ 'Ligne AILES, Société civile Synchrotron SOLEIL, 91192 Gif sur Yvette, France \\ ${ }^{d}$ Radiochemistry Division, Bhabha Atomic Research Centre, Trombay, Mumbai - 400085, India \\ *Corresponding Authors: Email: sabyasachi.patra@cea.fr; spatra@barc.gov.in (S.P.) \\ sophie.le-caer@cea.fr (S.L.C.) \\ antoine.thill@cea.fr (A.T.)
}

\begin{abstract}
Photocatalytic production of fuels, even in small quantities, from the mineralization of hazardous pollutants, is a promising and renewable way of recycling wastewater. In the present work, the potential of methyl functionalized inorganic aluminosilicate nanotubes (methyl imogolite/Imo$\mathrm{CH}_{3}$ ) as photocatalytic nanoreactors for this application is demonstrated. Using the phototoxic polycyclic aromatic hydrocarbon dibenzo(a,h)anthracene (DBAN) as a model pollutant, we show that DBAN molecules can be efficiently trapped inside $\mathrm{Imo}^{-} \mathrm{CH}_{3}$ nanotubes from an aqueous medium to undergo subsequent oxidative photo-degradation under UV light. The kinetics of this photo-degradation were shown to depend strongly on both the initial DBAN concentration in the nanotubes and the presence/absence of dissolved dioxygen. The photo-degradation process followed a complex mechanistic pathway, consisting of combined photo-oxidation and photocycloaddition reactions, where detection of carbon dioxide $\left(\mathrm{CO}_{2}\right)$ as a photo-oxidation product confirmed the mineralization of encapsulated DBAN. $\mathrm{CO}$ and $\mathrm{CH}_{4}$ molecules were also formed, however these could arise from the further photo-reduction of $\mathrm{CO}_{2}$ on the external surface of the nanotubes. Moreover, dihydrogen $\left(\mathrm{H}_{2}\right)$ was produced upon UV illumination under anaerobic conditions due to water reduction reactions on the external surfaces of $\mathrm{Imo}-\mathrm{CH}_{3}$ nanotubes. The
\end{abstract}


possible mechanistic pathways of these processes are proposed, and the dual capability of Imo$\mathrm{CH}_{3}$ nanotubes for simultaneous pollutant degradation and $\mathrm{H}_{2}$ production is then demonstrated $-\mathrm{a}$ rare feat for a single photocatalyst material.

Keywords: charge separation; photo-catalysis; confinement; imogolite; wastewater management; energy conversion

\section{Introduction}

Since the pioneering discovery of photocatalytic water splitting on $\mathrm{TiO}_{2}$ electrodes by Fujishima and Honda in 1972, ${ }^{1}$ the utilization of solar energy for various applications has been intensively studied with two central domains of interest: energy and environment. ${ }^{2-4}$ During the past few decades, tremendous efforts have been made to identify low cost and better performing photocatalytic platforms that are commercially practical, and a large number of semiconducting materials such as $\mathrm{TiO}_{2},{ }^{5} \mathrm{CdS},{ }^{6} \mathrm{CuS},{ }^{7} \mathrm{BiVO}_{4},{ }^{8} \mathrm{WO}_{3},{ }^{9} \mathrm{ZnO},{ }^{10} \mathrm{GaN},{ }^{11} \mathrm{ZnO}: \mathrm{GaN}$ solid solution, ${ }^{12}$ perovskites, ${ }^{13}$ and $\mathrm{g}-\mathrm{C}_{3} \mathrm{~N}_{4}{ }^{14}$ have been explored. One approach to achieve efficient solar conversion is to modify the base photocatalyst through the addition of dopants ${ }^{15}$ and/or co-photocatalysts, ${ }^{16}$ whether throughout the material or on its surface. However, the energy conversion efficiency of such photocatalysts to date has still not reached the expected threshold for commercial use,${ }^{17}$ and thus, the quest for new photocatalytic materials is ongoing.

Semiconducting materials with hollow nanostructures are particularly appealing in photocatalysis due to several advantages, such as enhanced light harvesting, better charge separation due to a reduced charge transfer distance across the thin wall of the material and higher rates of surface reactions due to increased surface areas. ${ }^{18}$ Additionally, the occurrence of polarization in a photocatalyst material can promote charge separation by facilitating the migration of electrons and holes in opposite directions. ${ }^{19}$ Recent theoretical predictions by Teobaldi and coworkers of the existence of a permanent polarization across the walls of imogolite nanotubes make it an increasingly attractive material for photocatalysis, i.e. one which processes the advantageous attributes of both hollowness and polarization. ${ }^{20,21}$ Very recently, the predicted effects of wall polarization in imogolite were experimentally proven by our group using radiolysis of confined water. $^{22}$

Imogolite is a natural aluminosilicate nanotube with the general formula $\mathrm{Al}_{2} \mathrm{O}_{3} \mathrm{Si}(\mathrm{OH})_{4}{ }^{23}$ These nanotubes are single-walled with a highly monodisperse external diameter of $2.6 \mathrm{~nm},{ }^{24,25}$ 
and are completely hydrophilic on the external and internal surfaces. They have a band gap around $5.2 \mathrm{eV},{ }^{26,27}$ due to which, electrons and holes are produced after the absorption of high-energy electromagnetic radiation. Their one-dimensional morphology may also favor the efficient diffusion of photo-generated electrons and holes. ${ }^{11,28-33}$ The structure being hollow with a thin wall, electrons are driven towards the external surface and the holes inside of the nanotubes. ${ }^{20-22}$ This property can be particularly interesting to the use of imogolite as a photocatalytic or cophotocatalytic nanoreactor for (reduction-oxidation) Redox reactions favoring the oxidation (resp. reduction) reaction inside (resp. outside) the nanocavity.

Recent studies have shown that the diameter, length and surface properties and reactivity of imogolite nanotubes can be tuned. ${ }^{34-43}$ Among the possible modifications, functionalization of the internal walls with alkyl groups is particularly appealing, where the nanotube cavity becomes hydrophobic and the external surface retains its hydrophilicity. ${ }^{44-47}$ Indeed, such methylfunctionalized imogolite nanotubes $\left(\mathrm{Imo}_{-} \mathrm{CH}_{3}\right)$ can capture small organic molecules very efficiently, ${ }^{48,49}$ making them a promising material to pre-concentrate organic pollutants from contaminated water. ${ }^{50}$ Importantly, this chemical modification of the internal surface does not prevent the formation of an intra-wall electric field. ${ }^{51}$ Therefore, the Janus character of $\mathrm{Imo}^{-\mathrm{CH}_{3}}$ nanotubes with an hydrophobic internal core and an hydrophilic external surface with strong wall polarization provides a unique opportunity to perform oxidative photo-degradation of encapsulated organic pollutants inside the nanotubes with simultaneous reduction reactions on the external surface.

In this work, we investigated the predicted curvature-induced charge separation in hybrid methyl-functionalized imogolite nanotubes using a photocatalytic Redox reaction relevant to the treatment of water contaminated with organic pollutants. As a model pollutant, we chose the highly phototoxic polycyclic aromatic hydrocarbon $(\mathrm{PAH})$, dibenzo(a,h)anthracene (DBAN) ${ }^{60} \mathrm{PAHs}$ are ubiquitous in environment and are of significant concern due to their carcinogenicity and toxicity, ${ }^{52}$ affecting primarily the lungs, ${ }^{53}$ bladder $^{54}$ and skin. ${ }^{55}$ These molecules are mainly formed by incomplete combustion of organic materials and are produced by various industries. The hazards of PAH increase upon exposure to visible or UV light, a phenomenon known as photo-toxicity. ${ }^{56-}$ ${ }^{58}$ Enzymatic metabolization of small PAH molecules is the most conventional way to convert them into easily eliminated water-soluble compounds, ${ }^{59}$ but larger PAHs such as DBAN, having at least four aromatic rings, are extremely resistant to enzymatic degradation. ${ }^{60-63}$ 
Taking advantage of the Janus character of the nanotubes and of the predicted curvatureinduced charge separation across the nanotube wall, the aims of the present work were to $(i)$ encapsulate relatively large DBAN molecules inside the $\mathrm{Imo}_{-} \mathrm{CH}_{3}$ cavity, (ii) study their possible degradation by photocatalytic oxidation and (iii) use the latter as a driving force to produce fuels such as dihydrogen $\left(\mathrm{H}_{2}\right)$. The encapsulation and loading concentration of DBAN in $\mathrm{Imo}^{-\mathrm{CH}_{3}}$ nanocavity was studied by UV-Vis absorption spectroscopy and small angle X-ray scattering (SAXS). Using combined UV-Vis and fluorescence spectroscopies, the photochemical reactions induced in DBAN encapsulated in aqueous solutions of $\mathrm{Imo}^{-\mathrm{CH}_{3}}$ were analyzed as a function of various experimental parameters (e.g. the nature of the gaseous atmosphere and the influence of the loading concentration) and compared to those obtained for DBAN in bulk hexane. The gaseous degradation products were measured along with the production of some valuable fuels, and a model for the photo-degradation and fuel production mechanism was proposed.

\section{Experimental}

\subsection{Materials}

Aluminum-tri-sec-butoxide (97\%), trimethoxymethylsilane (TMMS; 98\%), hydrochloric acid $(\mathrm{HCl} ; 37 \%)$, potassium bromide (FT-IR grade; $\geq 99 \%)$ and dibenzo(a,h)anthracene (DBAN; 97\%) were purchased from Sigma-Aldrich and used without further purification. Ultra high purity Ar $(99.9999 \%)$ and $\mathrm{O}_{2}(99.9999 \%)$ gas cylinders were purchased from Air Products. MilliQ water $\left(18.2 \mathrm{M} \Omega \cdot \mathrm{cm}\right.$ at $\left.25^{\circ} \mathrm{C}\right)$ was used throughout the present work.

\subsection{Synthesis of Imo- $\mathrm{CH}_{3}$ nanotubes}

Imogolite nanotubes with a hydrophobic internal cavity $\left(\mathrm{Imo}-\mathrm{CH}_{3}\right)$ were synthesized using a procedure inspired by Bottero et al. ${ }^{44}$ Briefly, aluminum-tri-sec-butoxide was added to a solution of hydrochloric acid $(75 \mathrm{mM})$ in the presence of trimethoxymethylsilane (TMMS). The molar ratios of $\mathrm{Al}: \mathrm{HCl}: \mathrm{Si}$ were fixed at 2:1:1.2. The mixture was initially turbid and became clear after vigorous stirring at ambient temperature for an hour. This solution was subsequently kept in an oven at 90 ${ }^{\circ} \mathrm{C}$ for 5 days. The resultant solution was slightly turbid and consisted of imogolite nanotubes as evidenced by their birefringence under cross-polarized light. The imogolite solution was further purified by dialysis (molecular weight cutoffs of 6-8 $\mathrm{kDa}$ ) against milliQ water until the

conductivity of the permeate solution was reduced to $4 \mu \mathrm{S} \mathrm{cm}^{-1}$ or less. Due to the hydrophilic external surface, Imo- $\mathrm{CH}_{3}$ nanotubes form a stable colloidal dispersion in water. 


\subsection{Encapsulation of DBAN in the $\mathrm{Imo}-\mathrm{CH}_{3}$ cavity}

DBAN $\left(\mathrm{C}_{22} \mathrm{H}_{14}\right.$, molecular weight of $\left.278.3 \mathrm{~g} \cdot \mathrm{mol}^{-1}\right)$ is a five-ring polycyclic aromatic hydrocarbon with extremely low solubility in water. ${ }^{64,65} \mathrm{DBAN}$ was mixed with $\mathrm{Imo}^{-\mathrm{CH}_{3}}$ in aqueous solution (Imo- $\mathrm{CH}_{3}$, concentration of $7.5 \mathrm{mg} \cdot \mathrm{mL}^{-1}$ ) and kept under continuous stirring. The progressive encapsulation of DBAN in $\mathrm{Imo}^{-\mathrm{CH}_{3}}$ nanotubes was followed regularly with a UV-Vis spectrophotometer (UB-2450, Shimadzu) and a HORIBA fluoromax-4 spectrofluorometer. A solution of pristine imogolite nanotubes of the same concentration was also kept under stirring and used as the reference for the UV-Vis spectroscopy measurements. In order to avoid the saturation of absorption and fluorescence intensity, the samples were diluted with Milli-Q water. A quantitative measure of the encapsulation was obtained from the increase in internal electron density of the nanotubes obtained from small angle X-ray scattering (SAXS) performed on a laboratory X-ray beamline (Xeuss 2.0). The distance between the sample and the detector was 45.6 $\mathrm{cm}$. For the SAXS measurements, the nanotube dispersions were introduced in sealed glass capillaries with a diameter of $1.5 \mathrm{~mm}$. The counting time was $2 \mathrm{~h}$ for each sample. Radial averaging of scattered X-rays was carried out to obtain plots of the scattering intensity as a function of the scattering wave vector $(q$ ), where $q$ is equal to $4 \pi \sin \theta / \lambda$ ( $\lambda$ being the incident wavelength and $2 \theta$ the scattering angle). The raw SAXS profiles were corrected using the scattering contributions of water, the empty capillary and the electronic background, respectively.

\subsection{Photochemical studies}

\subsubsection{UV illumination of aqueous solutions of DBAN encapsulated in $\mathrm{Imo}_{-} \mathrm{CH}_{3}$}

UV illumination of the various solutions was performed in a standard quartz cuvette with a stopper (optical path equal to $1 \mathrm{~cm}$ ) and by using a benchtop illumination chamber equipped with eight 15 W low pressure mercury lamps (OSRAM G15T8/OF, ozone free) emitting at $253.7 \mathrm{~nm}$. The incident photon intensity at $253.7 \mathrm{~nm}$ was measured by a potassium ferrioxalate actinometer solution ${ }^{66}\left(0.006 \mathrm{M}\right.$ ) and found to be $6.9 \times 10^{-5}$ Einstein $\cdot \mathrm{L}^{-1} \cdot \mathrm{s}^{-1}$ (equivalent to $4.15 \times 10^{19}$ photons. $\mathrm{L}^{-}$ ${ }^{1} \cdot \mathrm{s}^{-1}$ ). The uncertainty of actinometry measurement was found to be equal to $7.5 \%$. The average temperature of the solutions was $\sim 30^{\circ} \mathrm{C}$ and did not vary significantly during the illumination. The cuvette was taken out of the illumination box at regular time intervals. The corresponding UV-Vis absorption spectra and fluorescence spectra were recorded. UV illuminations were performed for DBAN loaded in $\mathrm{Imo}_{-} \mathrm{CH}_{3}$ solutions at three different loading concentrations $\left(9 \times 10^{-6}, 3.1 \times 10^{-5}\right.$ and $8.1 \times 10^{-4} \mathrm{~mol} \cdot \mathrm{g}^{-1}$ where the DBAN amount [mol] is expressed as a function of the imogolite 
quantity [in g]; as deduced from SAXS) and under different gaseous environments $\left(\mathrm{O}_{2}\right.$, air and $\left.\mathrm{Ar}\right)$. In the case of the experiments performed under $\mathrm{O}_{2}$ or $\mathrm{Ar}$, the solutions were first bubbled for 30 minutes with respective gases and the cuvette was immediately closed.

\subsubsection{UV illumination of DBAN dissolved in hexane}

In order to understand the specific role played by the polarized wall of $\mathrm{Imo}-\mathrm{CH}_{3}$ nanotubes on the photochemical reactions taking place in DBAN under alkyl environment (methyl groups on the internal wall of Imo- $\left.\mathrm{CH}_{3}\right)$, control experiments were performed using a DBAN solution $\left(1.1 \times 10^{-}\right.$

$\left.{ }^{5} \mathrm{~mol} \cdot \mathrm{L}^{-1}\right)$ in a nonpolar alkane (hexane). The experimental conditions were the same as those described in the previous section.

\subsubsection{Detection and measurement of gaseous degradation products}

$\mathrm{H}_{2}, \mathrm{CH}_{4}, \mathrm{CO}$ and $\mathrm{CO}_{2}$ gases were quantified by gas chromatography (Agilent $\mu$-GC-R3000, SRA instrument) using argon as a carrier gas. Before illumination, the samples, placed in a Pyrex glass ampule comprising a standard quartz cuvette bottom with a $1 \mathrm{~cm}$ optical path length, were degassed for $30 \mathrm{~min}$ by argon or dioxygen bubbling, depending on the required gaseous environment. They were then outgassed at approximately $5 \times 10^{-3}$ mbar and subsequently filled with 1.5 bar of argon or dioxygen, depending on the desired gaseous environment. This operation was repeated five times for each sample.

\section{Results and Discussion}

Imogolite nanotubes are made of a curved octahedral gibbsite-like $\mathrm{Al}(\mathrm{OH})_{3}$ layer on the external surface that is covalently linked to isolated silicon tetrahedron units $\left(\mathrm{SiO}_{3}(\mathrm{OH})\right)$ on the inner surface via mutually shared oxygen atoms (Figure 1a). ${ }^{51}$ In the case of methyl imogolite (Imo$\mathrm{CH}_{3}$ ), the hydroxyl group in the silicon tetrahedral unit on the internal surface is replaced by a methyl group $\left(\mathrm{SiO}_{3}\left(\mathrm{CH}_{3}\right)\right.$ ), making the internal surface hydrophobic (Figure 1b). The morphology of Imo- $\mathrm{CH}_{3}$ nanotubes is well characterized by various techniques such as FT-IR, SAXS and TEM, as extensively discussed in previous publications from our group. ${ }^{47-49,67}$ Similar characterizations have been performed for the Imo- $\mathrm{CH}_{3}$ nanotubes synthesized in the present work. The conventional and cryo-transmission electron microscopy (TEM and Cryo-TEM) images of the $\mathrm{Imo}^{-\mathrm{CH}_{3}}$ nanotubes, synthesized in this work, are given in Figure S1 (Electronic Supplementary Information). The conventional TEM image shows fibrous spatial arrangement of the $\mathrm{Imo}^{-} \mathrm{CH}_{3}$ 
nanotube bundles and the Cryo-TEM image shows the highly monodisperse single-walled Imo$\mathrm{CH}_{3}$ nanotubes.

(a)

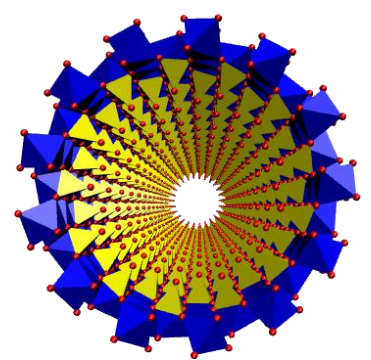

3D model of imogolite

(b)

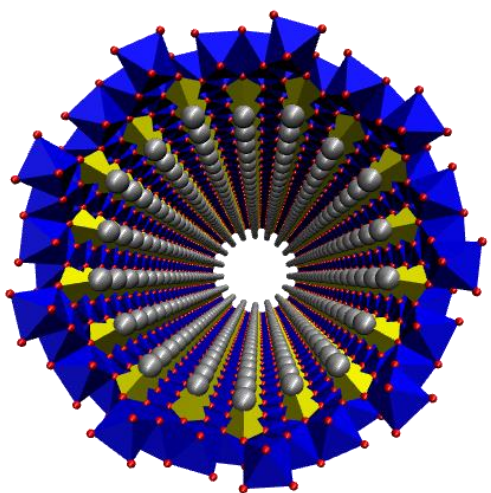

3D model of methyl imogolite $\left(\mathrm{Imo}_{\mathrm{m}} \mathrm{CH}_{3}\right)$ (c)<smiles>c1ccc2c(c1)ccc1cc3ccc4ccccc4c3cc12</smiles>

Structure of DBAN

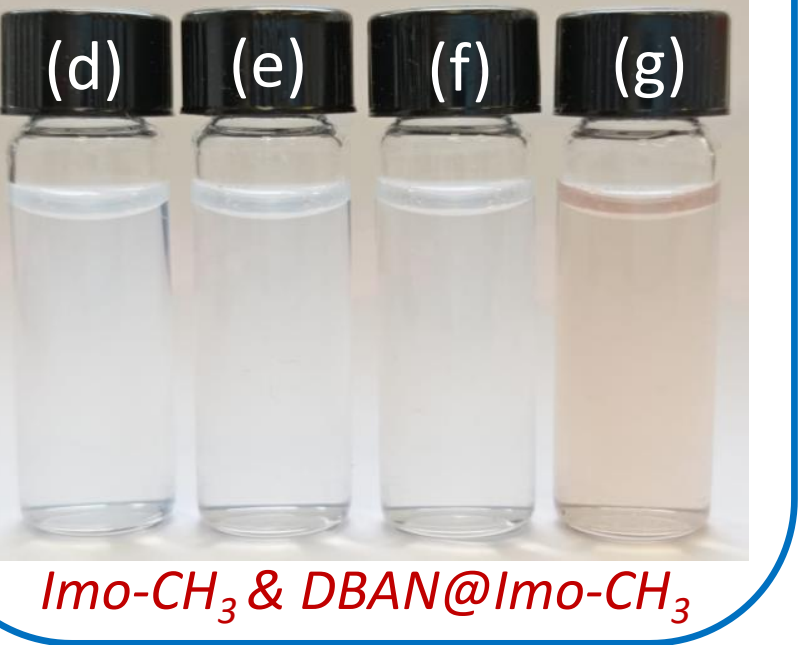

Figure 1. 3D model of natural imogolite (a) and methyl imogolite with hydrophobic core (b) [blue: aluminum octahedron; yellow: silicon tetrahedron; red: oxygen atom; grey: methyl group], chemical structure of DBAN (c), photographs of Imo- $\mathrm{CH}_{3}$ aqueous solution (7.5 g.L $\left.\mathrm{L}^{-1}\right)(d)$, and aqueous solution of varying concentrations of DBAN encapsulated in $\mathrm{Imo}_{-} \mathrm{CH}_{3}(e-g)$. The DBAN loading concentrations are: $9 \times 10^{-6}$ mol.g ${ }^{-1}(e), 3.1 \times 10^{-5}$ mol.g $g^{-1}(f)$ and $8.1 \times 10^{-4} \mathrm{~mol}_{\mathrm{g}} \mathrm{g}^{-1}(\mathrm{~g})$.

\subsection{Encapsulation of DBAN in Imo- $\mathrm{CH}_{3}$ nanotubes}

DBAN is a pale yellowish crystalline solid (see the chemical structure of DBAN in Figure 1c). Pristine Imo- $\mathrm{CH}_{3}$ solution is nearly transparent and colorless (Figure 1d). The slight turbidity is attributed to the residual presence of aluminum hydroxide as an impurity that could not be removed by dialysis. ${ }^{47}$ After equilibration of Imo- $\mathrm{CH}_{3}$ solutions with DBAN, the color of the solutions turn to orange as the DBAN concentration increases (Figure 1e-g). In order to confirm and quantify the 
encapsulation of DBAN within the hydrophobic nanocavity of the $\mathrm{Imo}^{-\mathrm{CH}_{3}}$ nanotubes, SAXS characterization of the solutions was carried out.

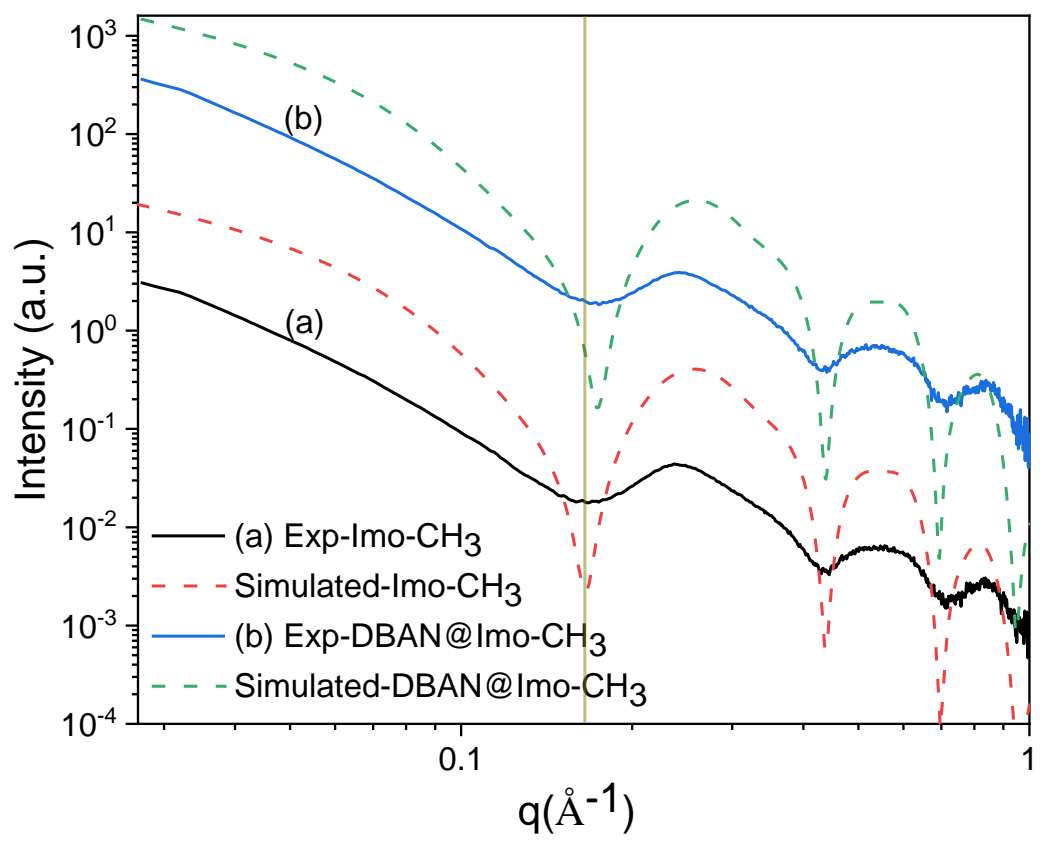

Figure 2. SAXS profiles of pristine Imo- $\mathrm{CH}_{3}($ a) and of DBAN encapsulated in Imo-CH3 (b) in aqueous solution (Imo- $\mathrm{CH}_{3}$ concentration is $7.5 \mathrm{mg} \cdot \mathrm{mL}^{-1}$ ). Dashed curves show the simulated SAXS profiles. The vertical line demonstrates the shift of the first intensity minimum to higher scattering vector upon loading, which confirms the encapsulation of DBAN inside the nanotubes. Comparison between curve (a) and (b) enables estimation of the amount of DBAN present inside the nanotubes.

Figure 2 displays the SAXS profiles of pristine $\mathrm{Imo}^{-\mathrm{CH}_{3}}$ in aqueous medium (a) and of an aqueous solution of Imo- $\mathrm{CH}_{3}$ nanotubes stirred with an excess of DBAN for 15 days (b). Prior to SAXS investigation, the undissolved DBAN powder was removed from the solution by centrifugation. At high scattering vector $(q)$, the SAXS profile presents the characteristic oscillations of single walled $\mathrm{Imo}^{-\mathrm{CH}_{3}}$ nanotubes, evidencing the good quality of the nanotubes used in the present study. The position of the intensity minima at different $q$ values and the characteristic deformation of the first oscillation confirm the alkyl functionalization of the Imo$\mathrm{CH}_{3}$ nanotubes. ${ }^{48}$ Further, the shift of the first minimum to higher scattering vector (highlighted by the vertical line in Figure 2) confirms the encapsulation of DBAN inside the nanotube cavities, as also evidenced by the increase in the internal electron density of the nanotubes (see also Table S1; Electronic Supplementary Information). ${ }^{48}$ Table S1 summarizes the parameters used for the 
simulated SAXS profiles of pristine and DBAN-loaded Imo- $\mathrm{CH}_{3}$ nanotubes. The encapsulation of DBAN in Imo- $\mathrm{CH}_{3}$ nanotubes was also evidenced by infrared spectroscopy as discussed in Electronic Supplementary Information (Figures S2, S3 and Table S2). Noteworthily, the infrared intensity of the different vibrational bands of DBAN seems to be significantly reduced upon encapsulation in Imo- $\mathrm{CH}_{3}$ nanotubes. This may be attributed to the dielectric screening by the polar Imo- $\mathrm{CH}_{3}$ wall, as already observed for molecules encapsulated in carbon nanotubes. ${ }^{68}$ The detection/quantification of DBAN encapsulated in $\mathrm{Imo}^{-\mathrm{CH}_{3}}$ nanotubes was also tested thanks to elution with acetone, followed by gas chromatography coupled to mass spectrometry analysis (GCMS). However, the high sublimation/boiling point (the boiling point of DBAN being equal to 524 ${ }^{\circ} \mathrm{C}$ ) and the low vapor pressure of DBAN ruled out the feasibility of elution and of the GC-MSbased quantification of DBAN in the present concentration range. Furthermore, the complete elution of DBAN from Imo- $\mathrm{CH}_{3}$ cavity using acetone was found to be difficult. Therefore, a quantitative estimation of encapsulated DBAN by elution is challenging.

Since DBAN is an aromatic hydrocarbon, it exhibits intense characteristic UV-Vis absorption and fluorescence emission spectra in organic solvents. ${ }^{69}$ Figure 3 a shows the UV-Vis absorption spectrum of DBAN in hexane, a nonpolar aliphatic hydrocarbon. Multiple maxima in a single absorption band are attributed to electronic excitations in different vibrational sublevels. The UV-Vis absorption spectrum of DBAN in hexane can be roughly divided into two parts, consisting of two sets of maxima, labelled A-C (EES-I) and D-L (EES-II), and associated with different electronic excitation states, ${ }^{70}$ with the most intense absorption peak at $297 \mathrm{~nm}$ (Peak F, Figure 3a). The relative absorbance of different vibrational levels changes with the increase of the DBAN concentration in hexane (Figure S4, Electronic Supplementary Information), suggesting co-facial stacking ${ }^{71}$ between DBAN molecules at the highest concentrations. It is worth noting that the UVVis absorption spectrum of DBAN in hexane is very close to the spectrum measured in dimethylformamide (DMF), a polar organic solvent. ${ }^{69}$ In hexane, the molar extinction coefficient at $\lambda_{\max }\left(\varepsilon_{297 \mathrm{~nm}}\right)$ is found to be $1.52 \times 10^{5} \mathrm{~L} \cdot \mathrm{mol}^{-1} \cdot \mathrm{cm}^{-1}$ (Figure 3a), which is similar to the value measured in DMF $\left(1.5 \times 10^{5} \mathrm{~L} \cdot \mathrm{mol}^{-1} \cdot \mathrm{cm}^{-1}\right) .{ }^{69}$

The increase in electron density in DBAN-loaded tubes, as compared to pristine $\mathrm{Imo}^{-\mathrm{CH}_{3}}$ ones (Figure 2), can be used to estimate the amount of trapped organic molecules. However, the internal electronic density of the pristine nanotubes is not equal to zero, which suggests that they 
are initially occupied by some residues of the synthesis such as alcohols. Therefore, DBAN encapsulation may lead to the exclusion of these small molecules from the nanocavity. This suggests that our calculation underestimates the actual DBAN loading concentration in the nanotubes. However, it gives a reasonable order of magnitude of this value. Using it and the corresponding UV-Vis absorption spectrum, the molar extinction coefficient $(\varepsilon)$ of DBAN encapsulated in Imo- $\mathrm{CH}_{3}$ suspensions could be calculated (see Electronic Supplementary Information for details of calculation). It was found to be $1.29 \times 10^{4} \mathrm{~L} \cdot \mathrm{mol}^{-1} \cdot \mathrm{cm}^{-1}$ at peak $\mathrm{F}$, which is roughly ten times lower than in hexane. Knowing the molar extinction coefficient, the loading concentration (in $\mathrm{mol} \cdot \mathrm{g}^{-1}$ ) of DBAN in Imo- $\mathrm{CH}_{3}$ suspensions could then be systematically obtained.

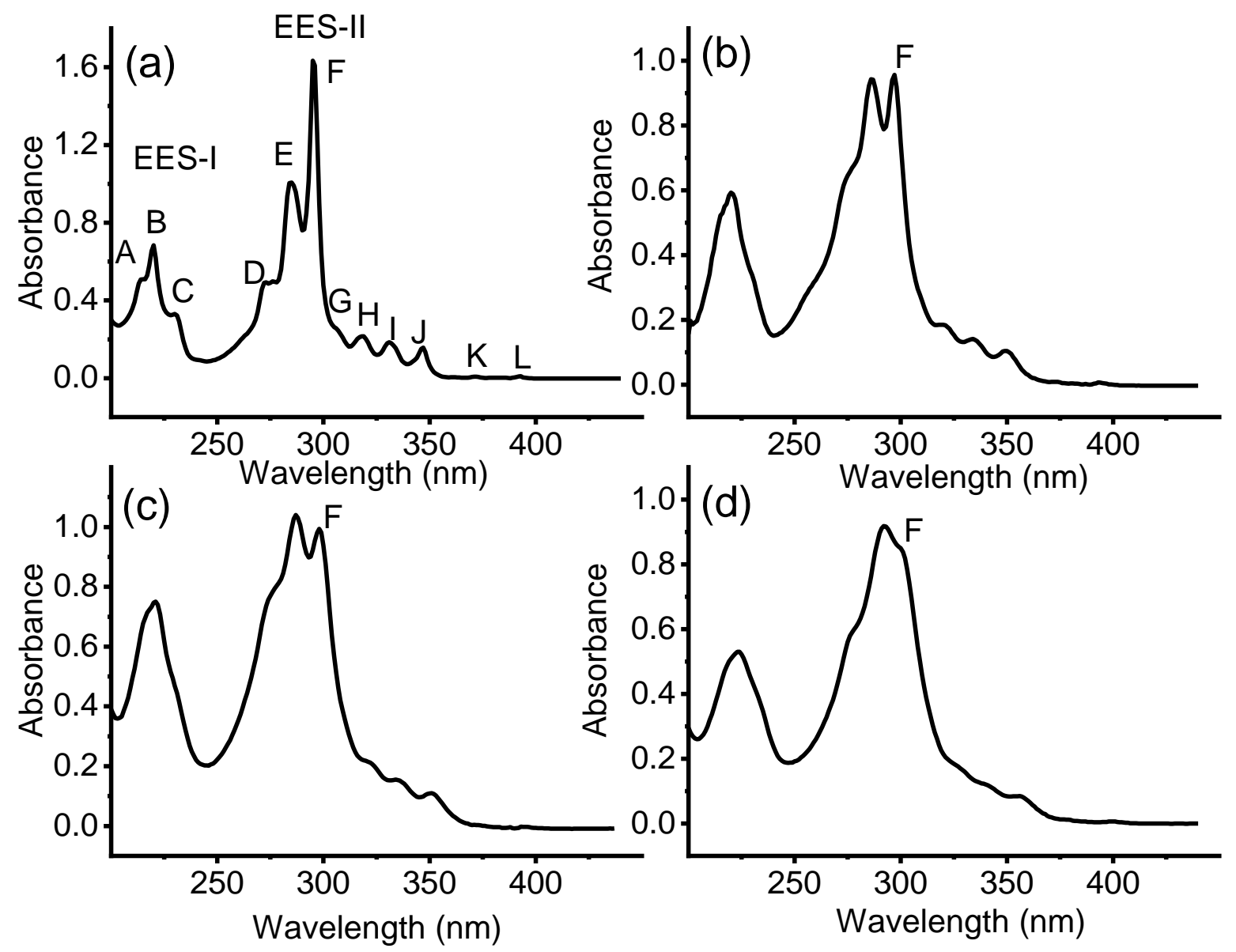

Figure 3. UV-Vis absorption spectrum of $D B A N\left(1.1 \times 10^{-5} \mathrm{~mol} \cdot \mathrm{L}^{-1}\right)$ in hexane (a), and of DBAN encapsulated in Imo- $\mathrm{CH}_{3}$ suspensions $(b-d)$. The DBAN loading concentrations were: $9 \times 10^{-6}$ $\mathrm{mol} \cdot \mathrm{g}^{-1}(\mathrm{~b}), 3.1 \times 10^{-5} \mathrm{~mol} \cdot \mathrm{g}^{-1}(\mathrm{c})$ and $8.1 \times 10^{-4} \mathrm{~mol} \cdot \mathrm{g}^{-1}(\mathrm{~d})$. The absorbance of the DBAN-loaded Imo- $\mathrm{CH}_{3}$ solutions $(b-d)$ was adjusted close to unity after dilution with ultra-pure water. The dilution factors were 1, 3 and 50 for $b, c$ and d, respectively. EES-I and EES-II correspond to two 
different electronic excitation states. Multiple maxima in EES-I and -II are attributed to electronic excitations in different vibrational sublevels.

The UV-Vis spectra of DBAN encapsulated in aqueous $\mathrm{Imo}_{-} \mathrm{CH}_{3}$ solutions are displayed in Figures 3(b-d) for various loading concentrations. The relative loading concentration of DBAN in these three samples was 1, 3 and 90, respectively. Roughly, these spectra share some common features with that of DBAN in hexane (Figure 3a). However, the absorption peaks shown in Figures 3(b-d) are less structured than those measured in hexane. This phenomenon is even more obvious as the DBAN concentration increases. Some slight modifications in the relative intensities of peaks $\mathrm{E}$ and $\mathrm{F}$, and in the position of the different maxima are reported in Figure S5 and in Table S3, respectively, in Electronic Supplementary Information.

Interesting information was also obtained from fluorescence spectroscopy measurements. Similar to the UV-Visible spectra, the fluorescence emission spectra of DBAN encapsulated in Imo- $\mathrm{CH}_{3}$ solutions were broader than the spectrum measured in hexane (Figure 4) and with a change in relative intensities (Figure S6, Electronic Supplementary Information). Moreover, a significant bathochromic shift of the peak maxima (Table S4, Electronic Supplementary Information) was also observed upon encapsulation. Fioressi et al. studied the absorption and fluorescence spectra of benzopyrene (BP), a similar polycyclic aromatic hydrocarbon to DBAN, both in hexane and adsorbed on a silica surface. ${ }^{72}$ The authors also observed a spectral broadening with a bathochromic shift in the case of BP adsorbed on a surface. This was attributed to BP-surface interaction and to a reversible BP-BP clustering in the absence of any solvent. The same may be true in the case of DBAN. Thus, the detected broadening of bands when DBAN is encapsulated inside the nanotubes can be attributed to the DBAN-Imo- $\mathrm{CH}_{3}$ internal surface interaction. The observed bathochromic shift as a function of increasing DBAN loading concentration can be attributed to the formation of stacked DBAN aggregates within the nanotube cavity via DBAN-DBAN co-facial weak adsorption. This clustering effect is also corroborated by the evolution of the color of the DBANloaded Imo- $\mathrm{CH}_{3}$ solution, as it changes from colorless to orangish red upon loading (Figure 1e-g). This suggests a decrease in the HOMO-LUMO gap for the DBAN clusters, which is in agreement with the theoretical calculations of Fioressi et al. in the case of BP. ${ }^{72}$ 
The UV-Vis absorbance of DBAN saturated water is negligible due to the extremely low aqueous solubility of DBAN (Figure S11a; Electronic Supplementary Information). The fluorescence emission spectrum of DBAN in water, as shown by Lehto et al., ${ }^{73}$ was also different from that measured in organic solvents such as hexane (Figure 4a) or DMF. ${ }^{69}$ These differences are attributed to the presence of DBAN in the form of aggregates/clusters in aqueous medium due to its poor solubility. It is noteworthy that the solubility of DBAN is increased significantly in aqueous medium in the presence of $\mathrm{Imo}^{-} \mathrm{CH}_{3}$ nanotubes. Indeed, the nanotubes with the Janus surface character play a similar role to a micelle or to different organic supramolecular hosts such as cyclodextrin, cucurbit[n]uril, etc. and solubilize the otherwise insoluble DBAN molecules in the hydrophobic nanocavities. Although the nanotubes are dispersed in aqueous media, the DBAN molecules are in a polar organic environment ${ }^{49}$ due to the wall curvature with the methyl groups on the internal wall of Imo- $\mathrm{CH}_{3}$, and a negligible volume of water vapor may enter in the hydrophobic nanochannels. ${ }^{74}$ This explains why the UV-Vis absorption and the fluorescence emission spectra of DBAN encapsulated in $\mathrm{Imo}-\mathrm{CH}_{3}$ are similar to those measured in hexane. However, as already mentioned before, the spectral features of encapsulated DBAN tend to disappear with the increase of DBAN loading in the nanotubes, due to same type of clustering effects. Noteworthily, the exchange of encapsulated DBAN from the nanotubes to external water is expected to be negligible due to the extremely poor solubility of DBAN in water. Moreover, once encapsulated, the DBAN molecules may also undergo adsorption on the hydrophobic wall. Therefore, encapsulation and adsorption may be difficult to discriminate in the present system. Figure 5 qualitatively gives a picture of the relative molecular crowding (DBAN) inside the Imo$\mathrm{CH}_{3}$ nanotube cavity in three different loading concentrations. 


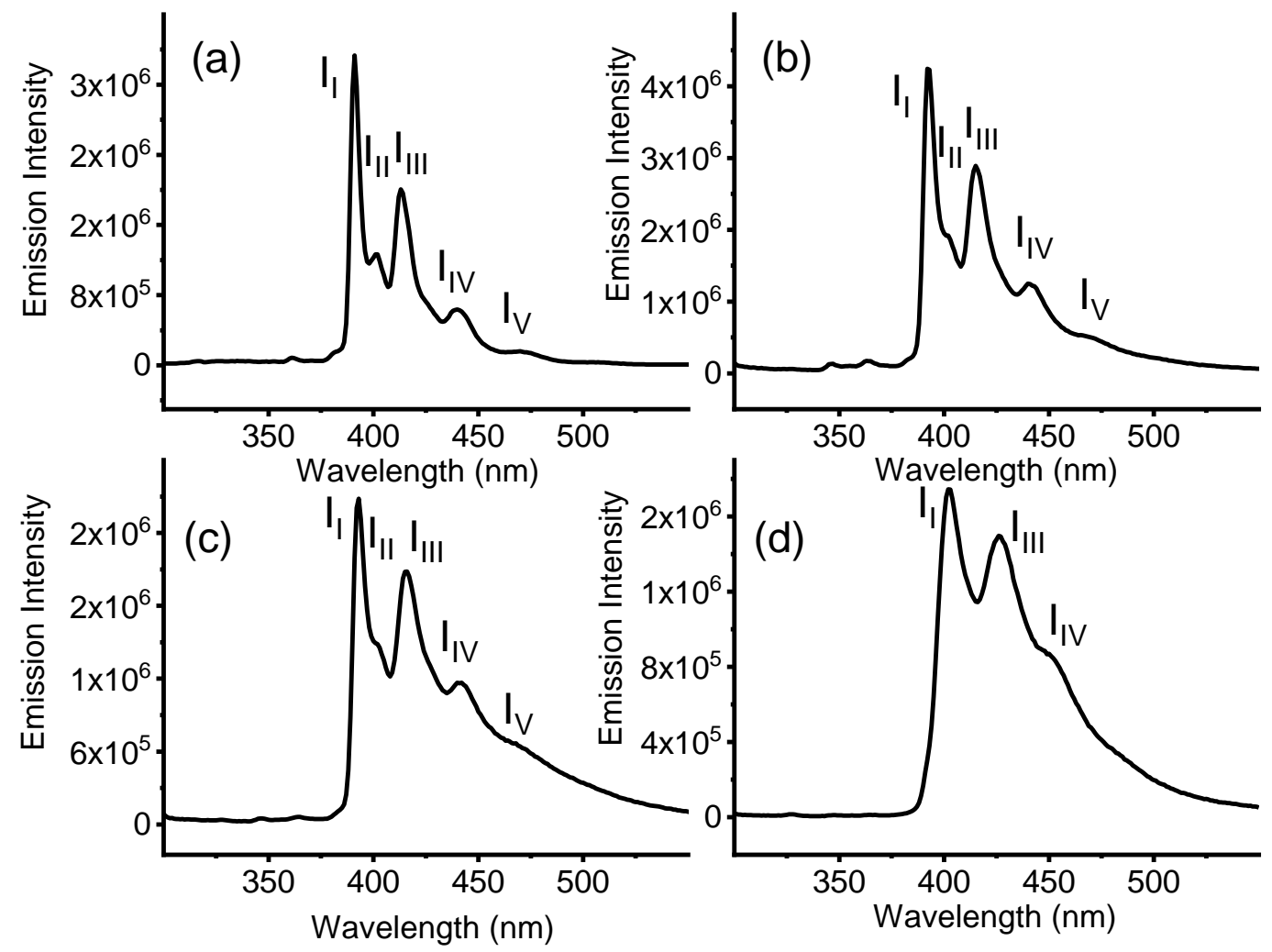

Figure 4. Fluorescence emission spectrum of DBAN $\left(1.1 \times 10^{-5} \mathrm{~mol} \cdot \mathrm{L}^{-1}\right)$ in hexane (a), and of DBAN encapsulated in Imo- $\mathrm{CH}_{3}$ in aqueous medium with a loading concentration of $9 \times 10^{-6} \mathrm{~mol} \cdot \mathrm{g}^{-}$ ${ }^{1}(\mathrm{~b}), 3.1 \times 10^{-5} \mathrm{~mol} \cdot \mathrm{g}^{-1}(\mathrm{c})$ and $8.1 \times 10^{-4} \mathrm{~mol} \cdot \mathrm{g}^{-1}(\mathrm{~d})$. All solutions were excited at $295 \mathrm{~nm}$. They were diluted with ultra-pure water to avoid the saturation of the fluorescence intensity. The dilution factors were 1, 3 and 50 for b, $c$ and d, respectively. Multiple maxima labelled as $I_{I}-I_{V}$ are ascribed to transitions involving different vibrational levels. 

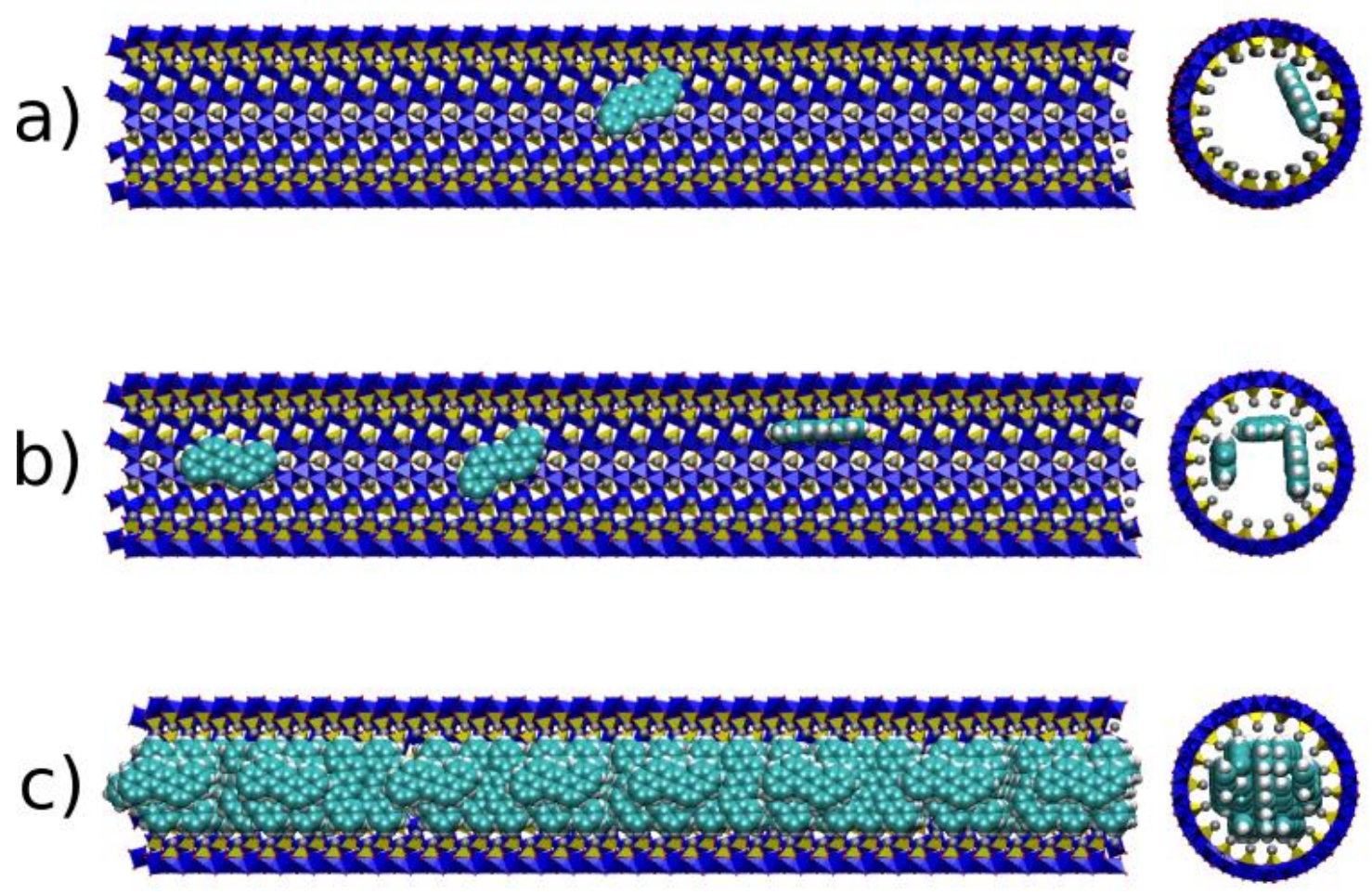

Figure 5. Schematic of the crowding of DBAN molecules in the Imo-CH3 nanotubes with a loading concentration of $9 \times 10^{-6} \mathrm{~mol} \cdot \mathrm{g}^{-1}(\mathrm{a}), 3.1 \times 10^{-5} \mathrm{~mol} \cdot \mathrm{g}^{-1}(\mathrm{~b})$ and $8.1 \times 10^{-4} \mathrm{~mol} \cdot \mathrm{g}^{-1}(\mathrm{c})$. It is important to note that the geometrical arrangements of DBAN molecules inside the tubes are not energetically optimized and are only for visual comparison of the molecular crowding at different loading concentrations.

\subsection{Photochemical studies}

\subsubsection{UV illumination of DBAN in hexane}

The internal wall of Imo- $\mathrm{CH}_{3}$ nanotubes provides an alkyl environment that is polarized due to the wall curvature. ${ }^{26,20,46,49}$ Hence, to isolate the surface effect of the polarized wall of Imo- $\mathrm{CH}_{3}$ on the photochemical reactions of DBAN, it is essential to understand these reactions in a bulk, nonpolar alkane environment. It is worth noting that there is very limited literature on the photolysis of DBAN in organic and aqueous media. Low et al. studied the photodegradation of various PAHs, including DBAN, in different organic solvents with varying polarity. ${ }^{75}$ They demonstrated that, under UV illumination with a $254 \mathrm{~nm}$ lamp, the half-life of DBAN in nonpolar hexane is 77 times 
longer than in a polar solvent such as methanol. This suggests that DBAN is resistant to photodegradation in non-polar or less polar environment. Deaerated solutions of PAHs in different organic solvents are known to form stable photo-dimers when exposed to UV light. ${ }^{76-83}$ Therefore, it is important to study the UV photolysis of DBAN in hexane under different gaseous environments.

Figure 6a shows the evolution of the UV-Vis absorption spectra of an air-saturated solution of DBAN in hexane upon illumination. The rapid decrease of the UV-Vis absorption intensity suggests a photo-cycloaddition reaction, as already shown for several anthracene derivatives. ${ }^{80}$ It corresponds here to the loss of the conjugated $\Pi$ system of DBAN due to the dimerization at the 7 and 14 positions of the DBAN structure (see Figure 1c). The decrease in absorbance is initially a slow process (Figure 6a), followed by a quick drop after roughly 40 minutes of illumination. It is worth noting that the "induction time" required for the absorbance value to drop suddenly is very dependent on the atmosphere: it is minimum when the sample is pre-bubbled with Ar, then increases when the sample is under air and further increases when it is pre-bubbled with dioxygen (Figure S7 in Electronic Supplementary Information). Therefore, dissolved dioxygen clearly plays an important role in this process. During the initial slow absorbance decrease phase, dissolved dioxygen in the organic solvent likely leads to the formation of unstable endoperoxides or hydroperoxide intermediates by a reversible reaction between DBAN and singlet dioxygen, thereby protecting the DBAN molecules against photo-cycloaddition. ${ }^{82,84}$ Consequently, the stage at which the rapid cycloaddition takes place refers to the moment at which dissolved dioxygen in the solution under illumination is fully depleted. It can be observed that, the relative intensity between the two most intense absorption peaks at 285 and $295 \mathrm{~nm}$ decreases during the "induction time" (see Figure 6a and Figure S8 in Electronic Supplementary Information). As discussed previously, stacking of DBAN molecules in hexane and in the Imo- $\mathrm{CH}_{3}$ cavity led to an increase in the relative absorbance between 285 and 295 nm (see Figure S4 in Electronic Supplementary Information and Figure 3). Hence, the decrease in relative absorbance $\left(\mathrm{Abs}_{285 \mathrm{~nm}} / \mathrm{Abs}_{295 \mathrm{~nm}}\right)$ during the "induction time" in Figure 6a and S8 (Electronic Supplementary Information) suggests de-stacking of DBAN molecules while depleting the dissolved dioxygen via the formation of endoperoxide / hydroperoxide derivatives, under UV illumination. 

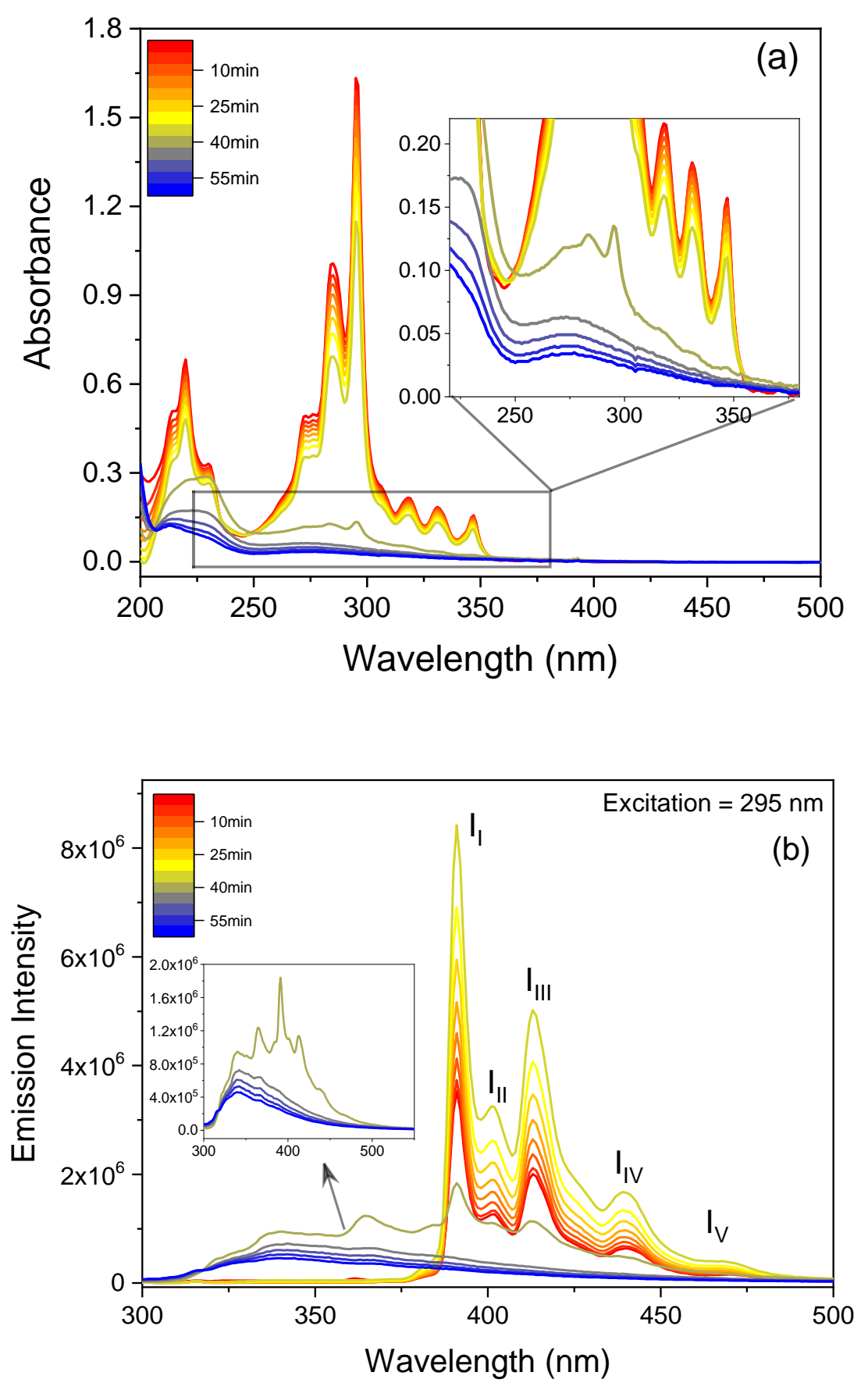

Figure 6. Evolution of UV-Vis absorption (a) and fluorescence emission (b) spectra of DBAN in hexane (initial concentration: $\left.1.1 \times 10^{-5} \mathrm{~mol} \cdot \mathrm{L}^{-1}\right)$ during illumination with UV light (253.7 $\mathrm{nm}$ ). For fluorescence measurements, the sample was excited at $295 \mathrm{~nm}$. The experiments were performed with air-saturated solutions in a stoppered quartz cell. 
This is supported by the evolution of the fluorescence emission spectra of the air-saturated solution of DBAN in hexane upon illumination (Figure 6b). One can observe that the intensity of the fluorescence spectra increases initially related to the molecular de-stacking of DBAN in hexane during the initial phase of photo-illumination, triggered by the formation of endoperoxide/hydroperoxide derivatives.

\subsubsection{UV illumination of DBAN encapsulated in $\mathrm{Imo}-\mathrm{CH}_{3}$ nanotubes in aqueous medium}

Lehto et al. have studied the photolysis of different PAHs in aqueous medium using fluorescence spectroscopy. They have demonstrated that all the PAHs, including DBAN, undergo photodegradation in aqueous media under illumination with a $254 \mathrm{~nm}$ UV light with an apparent first order rate constant ranging from $10^{-2}$ to $10^{-3} \mathrm{~min}^{-1} .^{73}$

Figure 7(a-c) presents the evolution of UV-Vis absorption spectra of DBAN in aqueous solutions of Imo- $\mathrm{CH}_{3}$ under UV illumination for various initial loading concentrations. Figure 7d displays the evolution of the corresponding fluorescence emission spectra at the lowest loading concentration. As already discussed in Section 3.1 (Figure 3), the UV-Vis absorption spectra of encapsulated DBAN were broad with modified relative intensities and a bathochromic shift as compared to the spectra of DBAN in hexane. This is due to DBAN-Imo- $\mathrm{CH}_{3}$ surface interactions and to DBAN-DBAN co-facial weak interactions. Under UV illumination, the UV-Vis absorption and fluorescence emission spectra of encapsulated DBAN evolved very differently (Figure 7) to that measured in bulk hexane (Figure 6). Indeed, steady changes in intensity were detected in all cases, except for very short illumination times for the highest concentration (Figure 7c). In this latter case (Figure 7c), the rapid decrease in absorption intensity after a short illumination time can be attributed to photo-cycloaddition of close DBAN molecules within the nanotube. This is similar to the processes observed for DBAN in hexane (Figure 6a). The absence of "induction time" in the case of encapsulated DBAN (Figure 7c) is possibly due to very high local concentration of DBAN inside the nanotubes and also to a lesser availability of dioxygen molecules inside the hydrophobic cavity. The fact that in all cases, the signals decay to zero at the highest illumination times suggests the photo-oxidation of DBAN molecules under UV irradiation, leading to the formation of

molecules that do not absorb in this spectral range or to gaseous products. The presence of isosbestic points (Figure 7(a-c)) suggests the formation of intermediate compounds during the 
photo-oxidation process of DBAN, absorbing around 240-245 $\mathrm{nm}$ and $400 \mathrm{~nm}$. Figure S9 (Electronic Supplementary Information) provides the temporal evolution of absorbance at different wavelengths. This evidences that the concentration of the intermediates goes through a maximum, before decreasing. These intermediates are finally converted to non-absorbing molecules or to gaseous products. Depending on the extent of nanotube loading concentration and on the gaseous atmosphere, the photochemical processes are complex and the photo-cycloaddition and photoredox phenomena may take place as collective non-separable events. As opposed to the photoredox reaction involving the charge pairs produced in $\mathrm{Imo}^{-} \mathrm{CH}_{3}$ nanotubes, the DBAN photocycloaddition reaction is promoted by UV light itself, without any charge transfer with the host nanotubes. It is worth noting that the concentration of intermediates or of the final non-gaseous molecules produced inside Imo- $\mathrm{CH}_{3}$ nanotubes was below the detection limit of conventional characterization techniques (FT-IR, ${ }^{1} \mathrm{H}$ NMR, etc.) and could not be characterized in the present work. However, a quantitative characterization of the gaseous products was possible by micro gas chromatography and is presented in Section 3.3. 

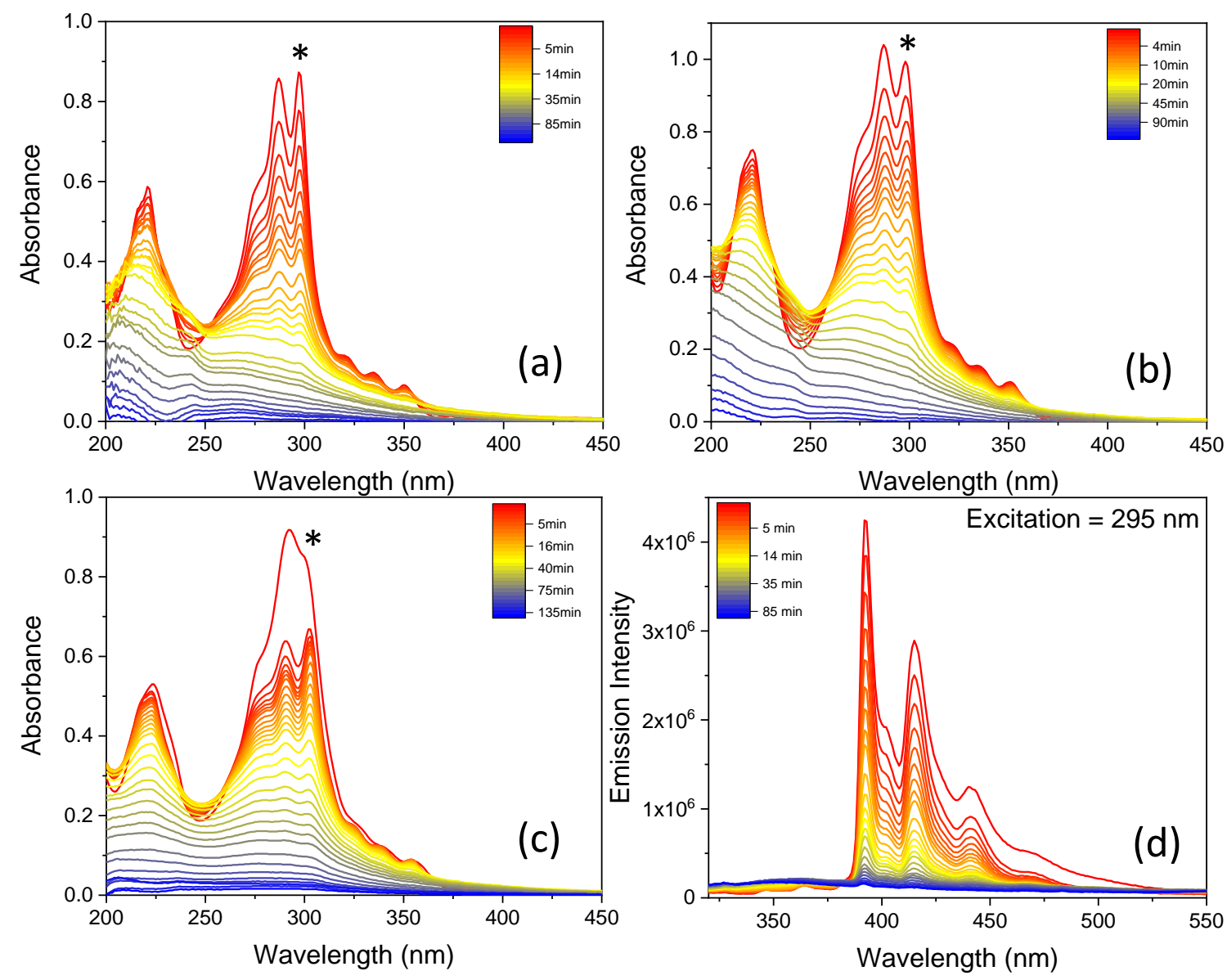

Figure 7. Evolution of $U V$-Vis absorption spectra of DBAN encapsulated in Imo-CH3 nanotubes in aqueous medium with an initial loading concentration of $9 \times 10^{-6} \mathrm{~mol} \cdot \mathrm{g}^{-1}(\mathrm{a}), 3.1 \times 10^{-5} \mathrm{~mol} \cdot \mathrm{g}^{-1}$ (b) and $8.1 \times 10^{-4} \mathrm{~mol} \cdot \mathrm{g}^{-1}$ (c) under illumination with UV light $(253.7 \mathrm{~nm})$. Evolution of the corresponding fluorescence emission spectra of DBAN encapsulated in Imo- $\mathrm{CH}_{3}$ nanotubes in aqueous medium with an initial loading concentration of $9 \times 10^{-6} \mathrm{~mol} \cdot \mathrm{g}^{-1}(\mathrm{~d})$. The experiments were performed in a stoppered quartz cell with air-saturated solutions. The asterisk (*) indicates peak $F$, located between 295 and $300 \mathrm{~nm}$.

Figure 8 shows the time variation of the value of the DBAN absorbance at peak $\mathrm{F}$ at time $t$ with respect to its value at time 0 (see Figure $3 \mathrm{a}$ for the nomenclature of DBAN absorption peaks), encapsulated in $\mathrm{Imo}_{-} \mathrm{CH}_{3}$ nanotubes in aqueous solution under UV illumination. No matter the loading concentration or atmosphere, the absorbance decreased to zero upon UV illumination. However, the decay shape depended on the loading concentration and could not be fitted with simple first or second order rate laws, suggesting a complex mechanism by superposition of the 
two reactions of photo-oxidation and photo-cycloaddition. Indeed, for the lowest loading concentration, the absorbance slowly decreased to zero. On the contrary, the sample with the highest loading concentration exhibited a rapid initial absorbance loss, followed by a much slower decay.

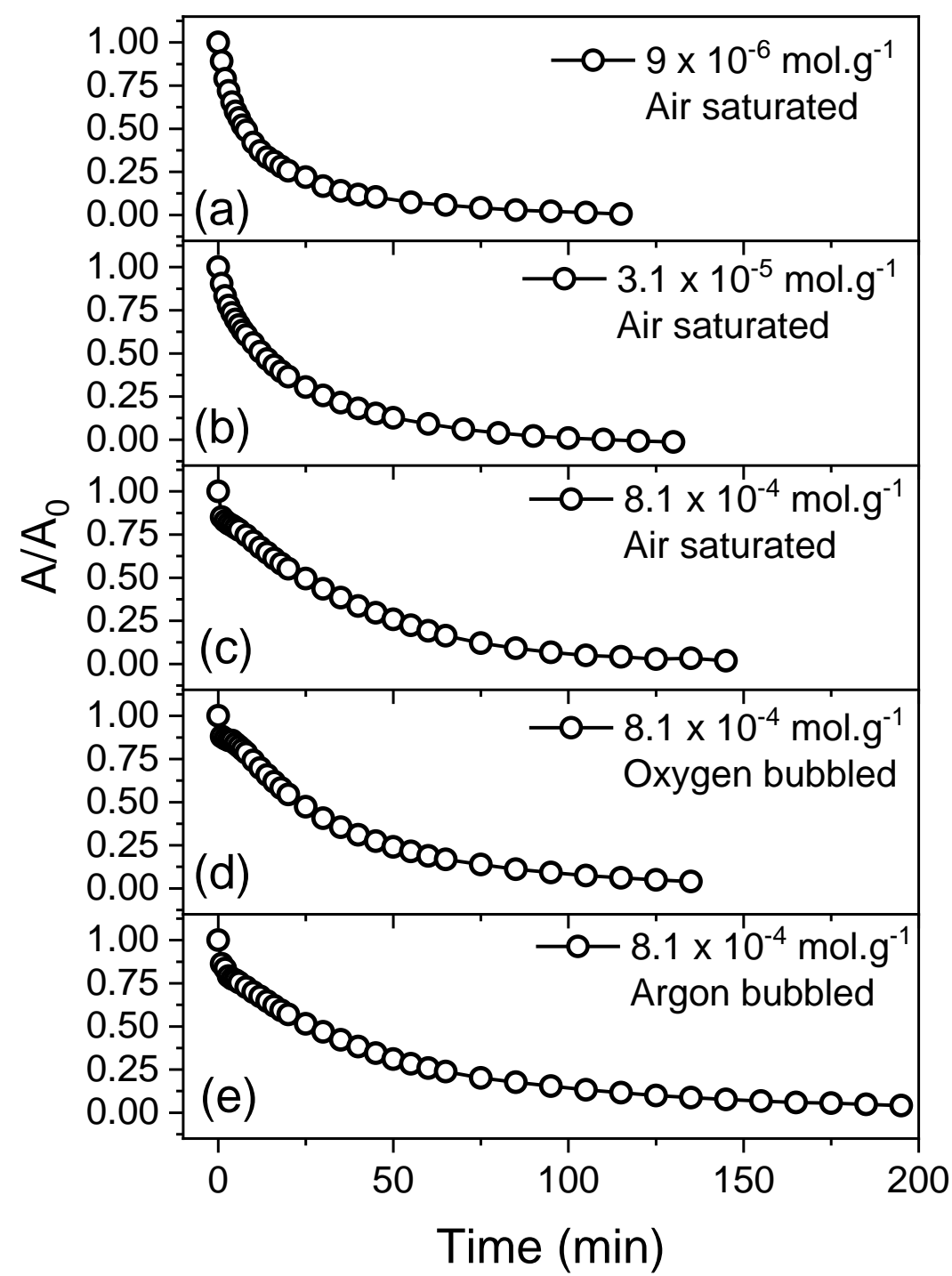

Figure 8. Kinetics of photo-oxidation and photo-cycloaddition (evolution of the absorbance of peak $F$, marked with an asterisk (*) in Figure 7 at time $t$ with respect to its value at time 0 ) of DBAN encapsulated in Imo- $\mathrm{CH}_{3}$ nanotubes in aqueous medium at different loading concentrations and gaseous atmospheres. Solid lines connecting the points are a guide to the eye. 
As seen in Figure 6a, the photo-cycloaddition of DBAN molecules in hexane under UV illumination also led to a drop of the UV-Vis absorbance. Hence, the loss of absorbance in Figures 7-8 may not be only due to the oxidation, but also to the concurrent and inevitable photocycloaddition of DBAN molecules under UV illumination. The fast initial decay of the absorbance in Figures 7-8 evidences the presence of two parallel phenomena such as photo-oxidation (first order) and photo-cycloaddition (second order) of DBAN within the nanotubes. At the highest concentration $\left(8.1 \times 10^{-4} \mathrm{~mol} \cdot \mathrm{g}^{-1}\right)$, the high molecular occupancy within the nanotube (almost 3 DBAN molecules per unit lattice imogolite ring) favors instantaneous cycloaddition of a significant fraction of encapsulated DBAN molecules within the nanotubes at the very beginning of the photochemical processes.

Obviously, the reaction mechanisms in question are very complex. However, we can obtain an estimate by simplifying the system and considering a first-order oxidation and a second-order cycloaddition as the two dominant phenomena that may be contributing to the loss of the UV-Vis absorbance of encapsulated DBAN under UV illumination. The decay rate of the DBAN concentration can then be expressed as:

$$
-\frac{d[D B A N]}{d t}=k_{1}[D B A N]+2 k_{2}[D B A N]^{2}
$$

where $k_{1}$ and $k_{2}$ represent the rate constants for the photo-oxidation and the photo-cycloaddition reactions, respectively. The solution of Equation 1 can be expressed as follows:

$$
\frac{1}{[D B A N]}=-\frac{2 k_{2}}{k_{1}}+\frac{2 k_{2}}{k_{1}} e^{k_{1}(t-C)}
$$

with $C$ a constant.

The rate constants $k_{1}$ and $k_{2}$ at varying loading concentrations and gaseous atmospheres, as summarized in Table S5 (Electronic Supplementary Information), have been obtained from the exponential fitting of $[D B A N]^{-1}$ vs. time plots as shown in Figure S10 (Electronic Supplementary Information). The good quality of the fit validates the proposed kinetic model. 
It is interesting to observe that the values of $k_{1}$ and $k_{2}$ depend on the experimental conditions. They vary with the DBAN loading and globally decrease when the DBAN loading increases, corresponding to a restriction in diffusion motions upon loading in the nanotubes. While $k_{1}$ is weakly dependent on the loading, $k_{2}$ strongly decreases upon loading. This can be attributed to the bimolecular character of the cycloaddition reaction. Table S5 (Electronic Supplementary Information) also shows that, as expected, $k_{1}$ depends on the presence/absence of dioxygen whereas $k_{2}$ does not. It is worth noting that even if $k_{2}$ decreases upon loading, $k_{2}[D B A N]^{2}$ increases upon loading (and the same for $k_{1}[D B A N]$ ). The accelerated kinetics are thus due to the large change in concentration, which largely compensates for the decrease in the rate constants.

As the presence/absence of dissolved dioxygen plays a role in the processes under consideration, photochemical studies of encapsulated DBAN were also carried out in solutions prebubbled with either dioxygen or argon prior to UV illumination (Figure 8d and 8e, respectively). In the case of the system pre-bubbled with $\mathrm{O}_{2}$, the extent of the decrease in relative absorbance during the initial cycloaddition phase (initial rapid decrease) was reduced. This suggests that gaseous $\mathrm{O}_{2}$ molecules enter the nanotube cavity and protect the internal DBAN molecules from photo-cycloaddition by forming endoperoxide/hydroperoxide intermediates in a similar mechanism to that described in Section 3.2.1. This makes photo-oxidation the dominant mechanism in this case. The photo-oxidation in the presence of dioxygen proceeds via the peroxide intermediates with a rate constant slightly lower than in air atmosphere. Conversely, for the system pre-bubbled with Ar, the external solution and the internal nanotube cavity do not contain $\mathrm{O}_{2}$. This causes the photo-cycloaddition to take place dominantly in the nanotube cavity within a short initial illumination.

Evidently, the kinetics of the photo-cycloaddition depend also on the diffusion coefficient of DBAN inside Imo- $\mathrm{CH}_{3}$ nanocavities. For example, Lopatkin et al. derived a kinetic model for the photo-dimerization of anthracene in a polymer matrix by considering the diffusion coefficient of anthracene molecules taking part in the dimerization reaction. ${ }^{85}$ In the present work, the diffusion of DBAN has not been considered separately for the sake of simplicity. However, the effect of diffusion is implicit from the dependence of $k_{2}$ on the loading concentrations. The weak dependence of $k_{1}$ on the loading concentration suggests that the presence of DBAN in monomeric or aggregated form (DBAN-DBAN co-facial stacking) has a weak influence on the kinetics of photo-degradation. 
This is in agreement with the conclusion of Kahan et al., ${ }^{86}$ who showed that the clustering of PAHs has no effect on the photo-degradation kinetics.

Table S5 (Electronic Supplementary Information) also summarizes the quantum yield of photo-oxidation of DBAN in $\mathrm{Imo}^{-} \mathrm{CH}_{3}$ cavity under varying loading concentrations and gaseous atmospheres. The quantum yield at a given wavelength is defined as the number of DBAN molecules undergoing hole $\left(\mathrm{h}^{+}\right)$mediated photo-oxidation per photon absorbed by $\mathrm{Imo}_{-} \mathrm{CH}_{3}$. The photo-oxidation quantum yield $\left(\phi_{1}\right)$ under the UV light source (peak wavelength at $253.7 \mathrm{~nm}$ ) was calculated using Equation 3. ${ }^{73,90,91}$

$$
\phi_{1}=\frac{k_{1}}{2.303 \times I_{\lambda, 0} \times \varepsilon_{I m o, \lambda} \times l}
$$

where $k_{1}$ represents the first order photo-oxidation rate constant $\left(\right.$ time $\left.^{-1}\right) ; I_{\lambda, 0}$ denotes the photon intensity at $253.7 \mathrm{~nm}\left(6.9 \times 10^{-5}\right.$ Einstein $\left.\cdot \mathrm{L}^{-1} \cdot \mathrm{s}^{-1}\right) ; \varepsilon_{I m o, \lambda}$ represents the molar absorptivity of the Imo- $\mathrm{CH}_{3}$ nanotube unit cell at $253.7 \mathrm{~nm}\left(1071.3 \mathrm{~L} \cdot \mathrm{mol}^{-1} \cdot \mathrm{cm}^{-1}\right)$, and $l$ is the cell path length $(1 \mathrm{~cm})$.

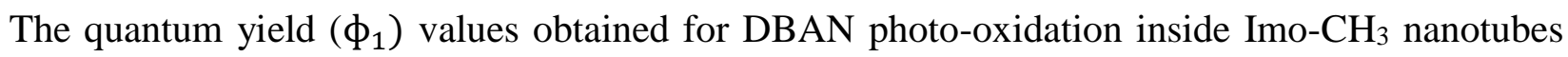
(Table S5; Electronic Supplementary Information) are similar or slightly higher (for the lowest loading concentrations) than the quantum yield of direct DBAN photolysis in water (approximately $\left.2.5 \times 10^{-3}\right) .{ }^{73}$ However, as the light source was nearly monochromatic and the band gap of Imo$\mathrm{CH}_{3}(\sim 5.2 \mathrm{eV})^{26}$ is slightly higher than the peak energy of the light $(4.9 \mathrm{eV}$ for a $253.7 \mathrm{~nm}$ wavelength), only a small fraction of the absorbed light was used to produce electrons and holes in Imo- $\mathrm{CH}_{3}$. However, it was revealed to be efficient enough to induce charge separation in $\mathrm{Imo}-\mathrm{CH}_{3}$ nanotubes. Also, the band gap of $\mathrm{Imo}-\mathrm{CH}_{3}$ may be reduced in presence of encapsulated DBAN due to the possible charge transfer from DBAN to $\mathrm{Imo}_{-} \mathrm{CH}_{3}$ nanotube, ${ }^{87-89}$ making the charge pair production by photo-excitation of imogolite more favorable. The comparison of UV-Vis absorption spectra of water saturated with DBAN (having a negligible UV-Vis absorption due to an extremely poor solubility) and of a DBAN-loaded Imo- $\mathrm{CH}_{3}$ aqueous solution (having a strong UV-Vis absorption characteristic of DBAN) evidences the encapsulation of DBAN inside the $\mathrm{Imo}^{-\mathrm{CH}_{3}}$ cavity (Figure S11, Electronic Supplementary Information). This further confirms that the quantum yield $\left(\phi_{1}\right)$ associated with the photolysis of the DBAN-loaded aqueous solution represents the photo-conversion of DBAN inside the $\mathrm{Imo}-\mathrm{CH}_{3}$ reactor. 


\subsection{Analysis of photo-degradation products}

Even if the loss of UV-Vis absorbance and fluorescence emission upon UV illumination of DBAN encapsulated in Imo- $\mathrm{CH}_{3}$ suggests the loss of conjugated double bonds in DBAN, it does not give any insight into its fate and into the possible production of gases. Therefore, various gases $\left(\mathrm{H}_{2}, \mathrm{CO}\right.$, $\mathrm{CO}_{2}$ and $\mathrm{CH}_{4}$ ) were identified and, if present, quantified by micro gas chromatography ( $\left.\mu-\mathrm{GC}\right)$. The sample with an initial loading concentration of $9 \times 10^{-6} \mathrm{~mol} \cdot \mathrm{g}^{-1}$ was illuminated for different times and the produced gases were measured. The experiments were performed under $\mathrm{O}_{2}$ or $\mathrm{Ar}$ atmosphere. After illumination under $\mathrm{O}_{2}$ atmosphere, $\mathrm{CO}_{2}$ and $\mathrm{CO}$ were detected by $\mu$-GC (Figure 9). The formation of $\mathrm{CO}_{2}$ suggests the mineralization of the encapsulated DBAN under UV illumination. It is worth noting that the illumination of the pristine $\mathrm{Imo}^{-\mathrm{CH}_{3}}$ solution also produced $\mathrm{CO}_{2}$ and $\mathrm{CO}$, but with a lower yield as compared to the DBAN-loaded Imo- $\mathrm{CH}_{3}$ sample. In the case of pristine Imo- $\mathrm{CH}_{3}$ suspension, the production of gases during illumination could be due to the photo-degradation of small alcohols inherently present as impurities in the cavity of the nanotubes. As the $\mathrm{CO}_{2}$ yield in the pristine Imo- $\mathrm{CH}_{3}$ sample did not increase significantly from 3 to 5 hours of illumination, the photo-degradation of the organic impurities can be assumed to be nearly completed in 3 hours. In the pristine sample, the saturation of the $\mathrm{CO}_{2}$ yield and the significantly low overall yield $\left(59.9 \mu \mathrm{mol} \cdot \mathrm{g}^{-1}\right)$ of carbon containing gases $\left(\mathrm{CO}_{2}+\mathrm{CO}\right)$ after 5 hours of UV illumination as compared to the carbon concentration in $\mathrm{Imo}^{-\mathrm{CH}_{3}}\left(5100 \mu \mathrm{mol} \cdot \mathrm{g}^{-1}\right)$ rule out the possibility of photo-degradation of the methyl groups inside $\mathrm{Imo}^{-\mathrm{CH}_{3}}$. The formation of a small concentration of $\mathrm{CO}$, under $\mathrm{O}_{2}$ atmosphere, suggests a further photo-reduction of $\mathrm{CO}_{2}$ (formed inside nanotube cavity) on the external surface of the nanotubes. In addition to $\mathrm{CO}$, trace amounts of $\mathrm{H}_{2}\left(0.48 \mu \mathrm{mol} \cdot \mathrm{g}^{-1}\right)$ and $\mathrm{CH}_{4}\left(1.35 \mu \mathrm{mol} \cdot \mathrm{g}^{-1}\right)$ were also detected after only 5 hours of illumination, which indicate that photocatalytic water reduction and $\mathrm{CO}_{2}$ conversion occurred on the external wall under the $\mathrm{O}_{2}$ atmosphere. When DBAN was encapsulated in the nanotubes, the overall yield of carbon containing gases $\left(\mathrm{CO}_{2}+\mathrm{CO}+\mathrm{CH}_{4}\right)$ produced after 5 hours of illumination (78.45 $\mu \mathrm{mol} \cdot \mathrm{g}^{-1}$; corrected from the gas production due to pristine $\mathrm{Imo}-\mathrm{CH}_{3}$ ) suggests that $40 \%$ of the initial DBAN concentration was transformed to small gaseous products after 5 hours. Clearly, the actual percentage of DBAN mineralization after 5 hours of illumination may be higher, as other possible gaseous and non-gaseous products, such as $\mathrm{HCOOH}, \mathrm{HCHO}, \mathrm{CH}_{3} \mathrm{OH}, \mathrm{C}_{2} \mathrm{H}_{4}, \mathrm{C}_{2} \mathrm{H}_{5} \mathrm{OH} \ldots$. 
might have also been formed during the $\mathrm{CO}_{2}$ conversion. However, they could not be detected by $\mu-\mathrm{GC}$ and gas chromatography coupled to mass spectrometry (GC-MS).

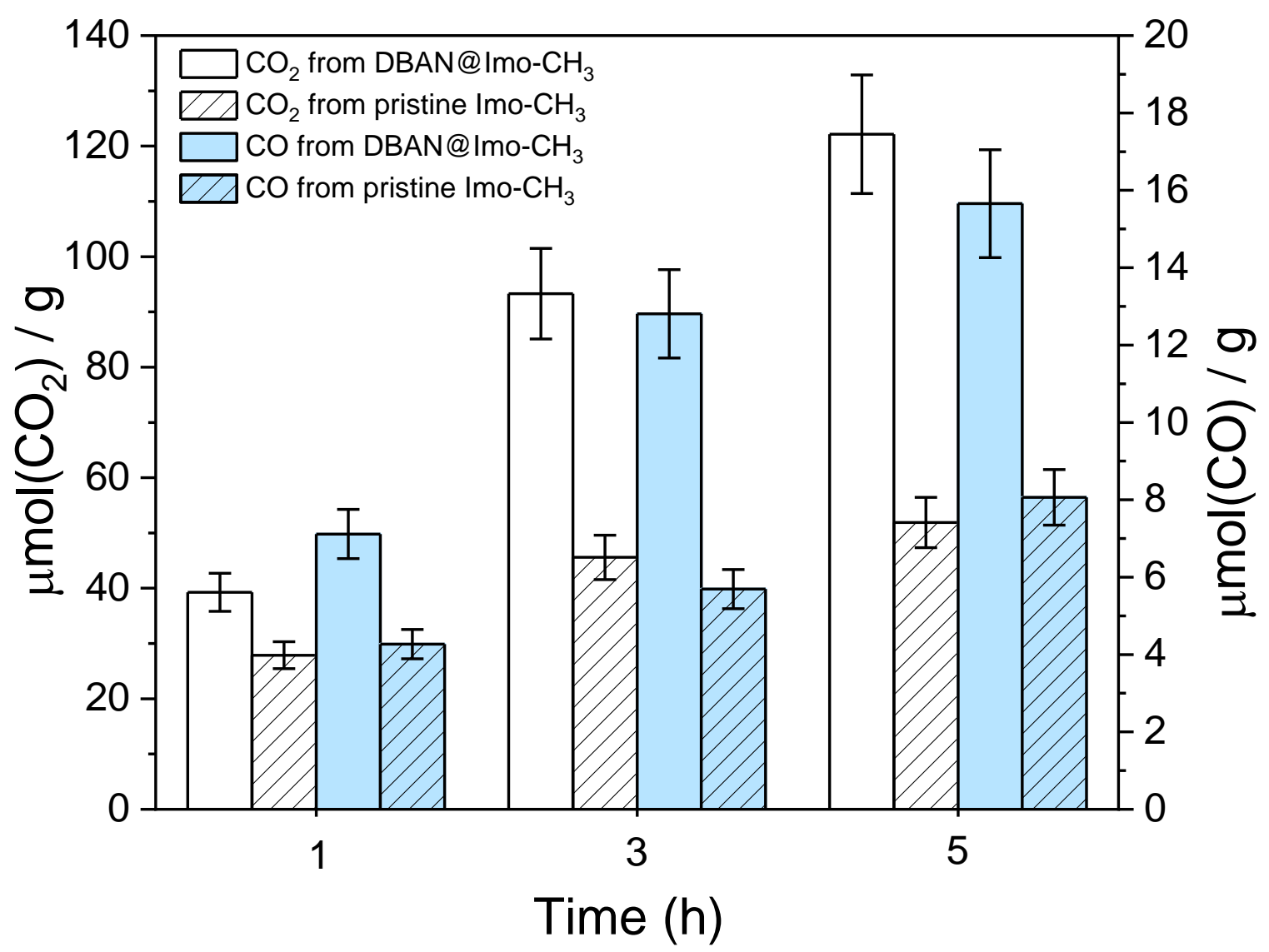

Figure 9. Amount of gases ( $\mathrm{CO}$ and $\mathrm{CO}_{2}$ ) produced during UV illumination of DBAN-loaded Imo$\mathrm{CH}_{3}$ aqueous suspension under $\mathrm{O}_{2}$ atmosphere. The amount of gases produced by illumination of pristine Imo- $\mathrm{CH}_{3}$ aqueous solution under identical conditions are also shown for comparison. The error bars are given on the figure. They include the uncertainty associated with $\mu-G C$ measurements (3.5 to $7.6 \%)$ and with the chemical actinometry (7.5\%).

The yields of $\mathrm{H}_{2}$ and $\mathrm{CH}_{4}$ from water and $\mathrm{CO}_{2}$ reduction, respectively, are significantly low under $\mathrm{O}_{2}$ atmosphere due to the electron scavenging by $\mathrm{O}_{2}$. These photo-reduction reactions are, in general, performed in deaerated solutions. ${ }^{92,93}$ On the contrary, photocatalytic degradation of organic compounds does not take place in the absence of dioxygen. ${ }^{94}$ Our spectroscopic studies provide a clue that the present photo-reactor can photo-oxidize the encapsulated DBAN molecules even without dioxygen (see Figure 8e and Figure S10e, Electronic Supplementary Information). In 
order to verify whether the present photo-reactor can degrade the encapsulated DBAN molecules even without dioxygen, specific gases $\left(\mathrm{CO}, \mathrm{CO}_{2}, \mathrm{CH}_{4}\right.$ and $\left.\mathrm{H}_{2}\right)$ were identified and, when present, quantified after illumination of DBAN-loaded $\mathrm{Imo}_{-} \mathrm{CH}_{3}$ suspensions under argon atmosphere (Figure 10). Interestingly, $\mathrm{CO}_{2}$ was produced, which suggests the oxidative mineralization of encapsulated DBAN under UV illumination, even without dioxygen. This is attributed to the exceptionally strong oxidizing potential of Imo- $\mathrm{CH}_{3}$ due to its wall curvature. ${ }^{20}$ Also, the $\mathrm{CO}_{2}$ conversion products such as $\mathrm{CO}\left(2 \mathrm{e}^{-}\right.$transfer $)$and $\mathrm{CH}_{4}\left(8 \mathrm{e}^{-}\right.$transfer $)$were detected in considerably larger amounts when the samples were under Ar atmosphere than under $\mathrm{O}_{2}$ atmosphere, suggesting further photo-reduction of $\mathrm{CO}_{2}$ on the external surface of the nanotube by the conduction band electrons $\left(e_{C B}^{-}\right){ }^{27,20}$ Remarkably, it is then possible to couple these two otherwise incompatible reactions, i.e, pollutant degradation and $\mathrm{CO}_{2}$ conversion, to produce valuables from wastewater. In addition to the production of carbon-containing gases, dihydrogen $\left(\mathrm{H}_{2}\right)$ was also produced (Figure 10). This demonstrates that in the absence of dissolved $\mathrm{O}_{2}$, water molecules can also act as electron acceptors and undergo two electron transfer reduction reactions by receiving conduction band electrons $\left(e_{C B}^{-}\right)$on the external surface of $\mathrm{Imo}_{-} \mathrm{CH}_{3}$ nanotubes. The photocatalytic degradation of encapsulated DBAN in absence of dioxygen and the simultaneous production of $\mathrm{H}_{2}$ and $\mathrm{CH}_{4}$ demonstrate the unique role of $\mathrm{Imo}_{-} \mathrm{CH}_{3}$ nanotubes to act as dual-photocatalytic nanoreactors for the simultaneous degradation of pollutants and production of fuels. These results suggest that by using the Janus-type Imo- $\mathrm{CH}_{3}$ photocatalytic reactor, organic pollutants could be successfully used as sacrificial agents for the production of dihydrogen and methane, avoiding the need of using electron donors such as organic acids and alcohols. ${ }^{95}$ Similarly, the trace organic impurities in the pristine Imo- $\mathrm{CH}_{3}$ cavity led to production of $\mathrm{CO}_{2}$ and other conversion products such as $\mathrm{CO}$ and $\mathrm{CH}_{4}$ under UV illumination with an Ar gaseous atmosphere (Figure 10). Note, a heat treatment to fully remove these organic impurities from Imo- $\mathrm{CH}_{3}$ could not be applied, as this may destroy the methyl functional groups. The overall yield of carbon-containing gases $\left(\mathrm{CO}_{2}+\mathrm{CO}+\mathrm{CH}_{4}\right)$ produced under argon atmosphere $\left(33.7 \mu \mathrm{mol} \cdot \mathrm{g}^{-1}\right.$; corrected from the gas production due to pristine Imo- $\mathrm{CH}_{3}$ ) suggests that $17 \%$ of the initial DBAN concentration was transformed to those small gaseous products after only 5 hours of illumination. 


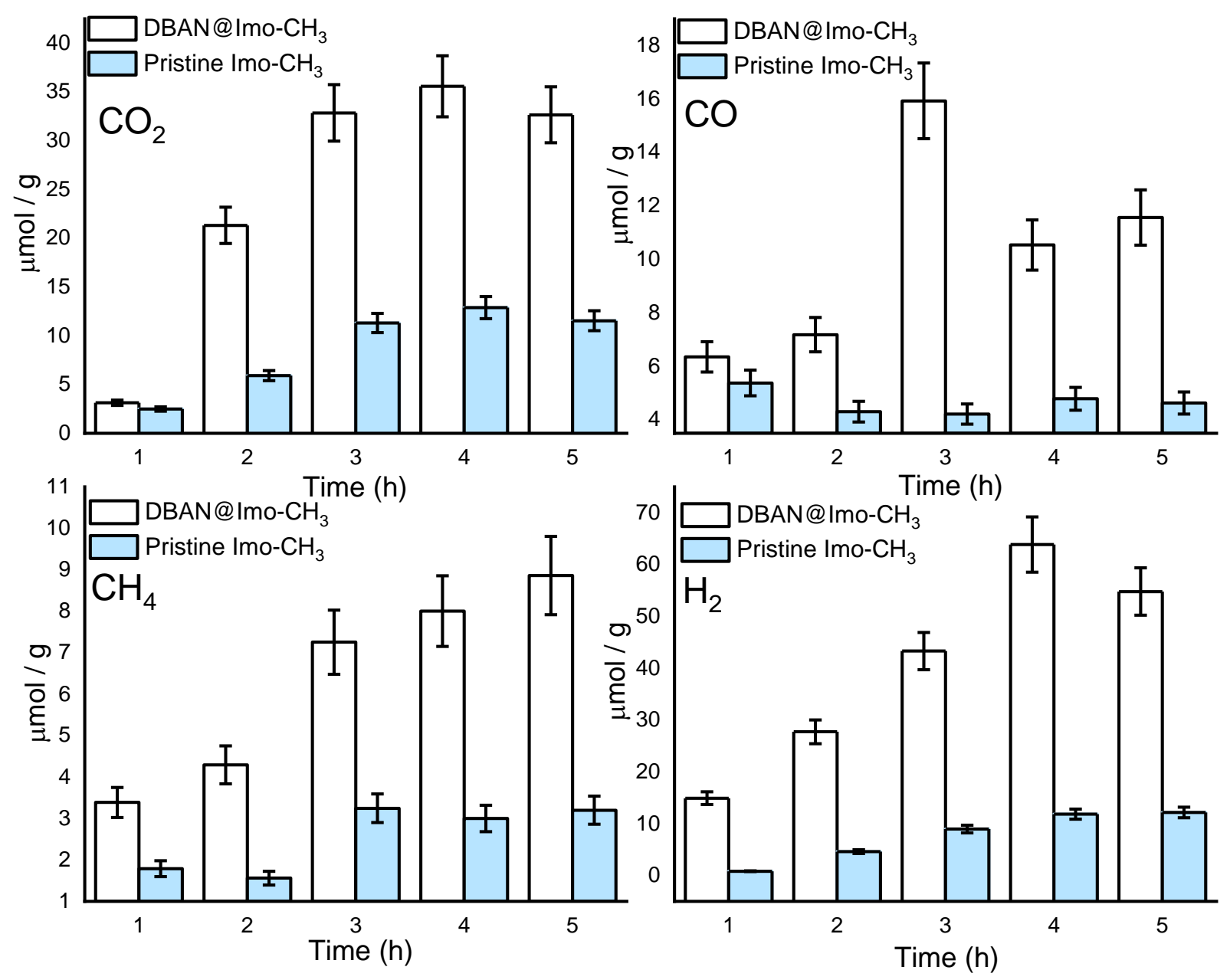

Figure 10. Evolution of the concentration of various gases $\left(\mathrm{CO}_{2}, \mathrm{CO}, \mathrm{CH}_{4}\right.$ and $\left.\mathrm{H}_{2}\right)$ produced during $U V$ illumination of DBAN-loaded Imo- $\mathrm{CH}_{3}$ and pristine Imo- $\mathrm{CH}_{3}$ solutions under argon atmosphere. The error bars are given on the figure. They include the uncertainty associated with $\mu$-GC measurements (3.5 to $7.6 \%$ ) and with the chemical actinometry (7.5\%).

The ability of Imo- $\mathrm{CH}_{3}$ nanotubes for $\mathrm{CO}_{2}$ and $\mathrm{H}_{2} \mathrm{O}$ reduction reactions can be corroborated qualitatively by comparison of the energy band edges of $\mathrm{Imo}^{-\mathrm{CH}_{3}}$ with the redox potentials of various couples. So far, the band diagram of imogolite has not been reported experimentally. In contrast and as already mentioned before, the band structure of the material has been theoretically calculated ${ }^{26}$ and the band gap energy value has been reported as being equal to $5.2 \mathrm{eV}$. The effect of defects on the band structure of the material has also been studied by DFT calculations. ${ }^{27}$ Even if the exact energy band edges of $\mathrm{Imo}-\mathrm{CH}_{3}$ is not known, it can be predicted using the well-known 
Matsumoto's correlations. ${ }^{96}$ Indeed, Matsumoto established linear correlations between band gap energy value and band edges for a variety of semiconductor oxides. With a band gap energy value $\left(\mathrm{E}_{\mathrm{g}}\right)$ of $\mathrm{Imo}-\mathrm{CH}_{3}$ equal to $5.2 \mathrm{eV}$, the conduction $\left(\mathrm{E}_{\mathrm{c}}\right)$ and valence $\left(\mathrm{E}_{\mathrm{v}}\right)$ band edges of $\mathrm{Imo}^{-\mathrm{CH}_{3}}$ nanotubes are calculated to be equal to $-1.37 \mathrm{~V}$ and $3.83 \mathrm{~V}$ vs. NHE, respectively. Obviously, the proton-assisted multi-electron $\mathrm{CO}_{2}$ reduction steps ${ }^{97,98}$ such as $\mathrm{CO}_{2} / \mathrm{HCOOH}(-0.61 \mathrm{~V}$ vs. NHE at $\mathrm{pH} 7), \mathrm{CO}_{2} / \mathrm{CO}(-0.53 \mathrm{~V}$ vs. $\mathrm{NHE}$ at $\mathrm{pH} 7), \mathrm{CO}_{2} / \mathrm{HCHO}$ (-0.48 V vs. NHE at $\left.\mathrm{pH} 7\right), \mathrm{CO}_{2} / \mathrm{CH}_{3} \mathrm{OH}$ $(-0.38 \mathrm{~V}$ vs. $\mathrm{NHE}$ at $\mathrm{pH} 7), \mathrm{CO}_{2} / \mathrm{CH}_{4}(-0.24 \mathrm{~V}$ vs. $\mathrm{NHE}$ at $\mathrm{pH} 7)$, are thermodynamically allowed on the Imo- $\mathrm{CH}_{3}$ external surface due to the less negative redox potentials of these couples as compared to the conduction band edge of $\mathrm{Imo}^{-\mathrm{CH}_{3}}\left(\mathrm{E}_{\mathrm{c}}\right)$. Also, the water reduction/oxidation reactions ${ }^{99}$ such as $\mathrm{H}^{+} / \mathrm{H}_{2}(-0.41 \mathrm{~V}$ vs. $\mathrm{NHE}$ at $\mathrm{pH} 7), \mathrm{H}_{2} \mathrm{O} / \mathrm{O}_{2}(+0.83 \mathrm{~V}$ vs. $\mathrm{NHE}$ at $\mathrm{pH} 7), \mathrm{H}_{2} \mathrm{O} /{ }^{\circ} \mathrm{OH}$ $(+2.27 \mathrm{~V}$ vs. NHE at $\mathrm{pH} 7)$ are also thermodynamically allowed. Furthermore, and in order to confirm the photo-reduction of $\mathrm{CO}_{2}$ on the Imo- $\mathrm{CH}_{3}$ surface, a degassed aqueous solution of DBAN-loaded Imo- $\mathrm{CH}_{3}$ was pre-bubbled with ultra-pure $\mathrm{CO}_{2}$ and subjected to UV illumination for 2 hours. The gaseous atmosphere was argon. Interestingly, the $\mathrm{CO}$ and $\mathrm{CH}_{4}$ yields were found to increase by $20 \%$ as compared to the experiment with no $\mathrm{CO}_{2}$, while the $\mathrm{H}_{2}$ yield decreased by a factor of 3. This confirms that $\mathrm{CO}_{2}$ undergoes photo-reduction on the $\mathrm{Imo}^{-\mathrm{CH}_{3}}$ surface, alongside with $\mathrm{H}_{2}$ production by $\mathrm{H}_{2} \mathrm{O}$ photo-reduction. The added $\mathrm{CO}_{2}$ molecules scavenge the photoelectrons due to reduction on the external surface of $\mathrm{Imo}_{-}-\mathrm{CH}_{3}$, thereby reducing the $\mathrm{H}_{2}$ yield. Obviously, some other gaseous/non-gaseous $\mathrm{CO}_{2}$ reduction products, that could not be detected by $\mu$-GC, may have also been formed.

Moreover, the $\mathrm{Imo}^{-\mathrm{CH}_{3}}$ photo-reactor has been found to be highly stable under high-energy UV illumination, as evidenced by UV-Vis spectroscopy and FT-IR characterization of the nanotubes before and after illumination (Figures S12-S13; Electronic Supplementary Information).

\subsection{Possible mechanisms of DBAN photo-degradation inside the $\mathrm{Imo}^{-\mathrm{CH}_{3}}$ photo-reactor}

The photo-oxidation mechanisms of DBAN inside the $\mathrm{Imo}^{-\mathrm{CH}_{3}}$ cavity are expected to be different from the mechanisms acting in bulk water. Due to the difficulty in fully characterizing the intermediates and any non-gaseous products produced in small quantities inside $\mathrm{Imo}^{-\mathrm{CH}_{3}}$ nanotubes, any possible mechanistic pathways are qualitatively discussed (Table 1). Upon absorption of photons with an energy higher than the band gap value of $\mathrm{Imo}^{-} \mathrm{CH}_{3}$, electron-hole 
pairs are produced across the wall of Imo- $\mathrm{CH}_{3}$ nanotubes (R1). These charges are predicted to be efficiently separated by the curvature-induced wall polarization. ${ }^{20-22}$ Nevertheless, the internal DBAN molecules are also excited (DBAN $\left.{ }^{*}\right)$ by UV radiation (R2). The excited DBAN molecules may be de-excited by several photophysical and energy transfer pathways (R3) or may undergo photo-cycloaddition to form dimers via the formation of excimers (R4) and subsequent deexcitation of the dimers (R5). The excited DBAN molecules may also undergo chemical reactions by hole/electron transfer reactions (R6). ${ }^{84}$ Using charge distribution calculations from density functional theory (DFT), Poli et al. have shown that the outer and inner surfaces of imogolite nanotubes are partially positively and negatively charged, respectively, which introduces a strong polarization across the nanotube wall. This suggests that the most probable reaction pathway for the DBAN or the excited DBAN molecules $\left(\mathrm{DBAN}^{*}\right)$ is an electron transfer to the $\mathrm{Imo}-\mathrm{CH}_{3}$ nanotube with the subsequent formation of a radical cation $\left(\mathrm{DBAN}^{+}\right)(\mathrm{R} 6)$. Owing to the high LUMO energy level of DBAN $(-1.49 \mathrm{eV}),{ }^{100}$ another de-excitation route could be the electron

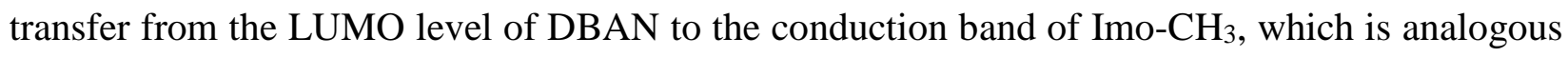
to dye-sensitized photocatalysts. ${ }^{101}$ Although this pathway is only energetically allowed if the conduction band edge of Imo- $\mathrm{CH}_{3}$ is lower in energy than the LUMO of DBAN. Unfortunately, the band structure of Imo- $\mathrm{CH}_{3}$ has been only predicted theoretically by DFT calculations until now, ${ }^{20,26}$ and an experimental validation of the band structure is needed to accurately compare the LUMO level of DBAN with the energy position of conduction band edge of $\mathrm{Imo}_{-} \mathrm{CH}_{3}$. Also, as the bottom of the conduction band arises from the electronic states of the hydroxyl moieties on the outer wall of Imo- $\mathrm{CH}_{3},{ }^{26}$ this de-excitation pathway of encapsulated DBAN is expected to be less probable than electron transfer to the top of the valence band (corresponding to electronic states located on the inner wall of $\mathrm{Imo}_{-} \mathrm{CH}_{3}$ ). ${ }^{26}$ Besides, there may be a few water molecules (in vapor state) present inside the nanocavity which can undergo hole $\left(\mathrm{h}^{+}\right)$-mediated oxidation to form $\mathrm{O}_{2}$ $\left(\mathrm{E}^{0}=+0.83 \mathrm{~V}\right.$ vs. $\mathrm{NHE}^{99}$ at $\left.\mathrm{pH} 7\right)(\mathrm{R} 7)$ or $\cdot \mathrm{OH}$ radicals $\left(\mathrm{E}^{0}=+2.27 \mathrm{~V}\right.$ vs. $\mathrm{NHE}^{99}$ at $\left.\mathrm{pH} 7\right)(\mathrm{R} 8)$ inside the nanocavity. The formation of DBAN radical cations (DBAN ${ }^{+}$) inside $\mathrm{Imo}^{-} \mathrm{CH}_{3}$ may also attract water molecules into the hydrophobic nanocavity by possible interaction with the lone pair electrons on the oxygen atom of water molecules, and thereby, increase the yield of internal water oxidation. The holes in valence band $\left(h_{V B}^{+}\right)$and the $\mathrm{OH}$ radicals are indeed powerful oxidants. These highly reactive $\cdot \mathrm{OH}$ radicals can ensure complete mineralization (R20) of the encapsulated DBAN molecules (or of the intermediates formed by the hole $\left(h_{V B}^{+}\right)$-mediated oxidation of DBAN) 
by $\mathrm{C}-\mathrm{C}$ bond scission via stepwise $\cdot \mathrm{OH}$ radical adsorption and $\mathrm{H}$ abstraction pathway. ${ }^{102,103}$ Figure S14 (Electronic Supplementary Information) presents the possible mechanistic pathways for the mineralization of DBAN. Concurrent with the oxidation reactions inside, $\mathrm{O}_{2}$ or $\mathrm{H}_{2} \mathrm{O}$ molecules on the external surface of the nanotubes can trap the photo-generated electrons in the conduction band $\left(e_{C B}^{-}\right)(\mathrm{R} 12-19)$. The dioxygen reduction reaction on the external surface may proceed in multiple pathways involving electron transfer from the external surface of the nanotube and reactions with co-adsorbed water molecules. This may also lead to the formation of strong oxidants such as $\cdot \mathrm{OOH}$ and $\mathrm{OH}$ radicals (R17, R19). ${ }^{104-106}$ These radicals are likely to enter into the nanotube cavity and participate in the mineralization of the encapsulated DBAN (R20).

As a few gaseous dioxygen molecules could be present in the $\mathrm{Imo}^{-\mathrm{CH}_{3}}$ cavity under equilibrium, in addition to the direct electron transfer from DBAN/DBAN* to Imo- $\mathrm{CH}_{3}$, another important pathway could be the formation of highly reactive singlet dioxygen $\left({ }^{1} \mathrm{O}_{2}\right)$ by energy transfer between $\mathrm{DBAN}^{*}$ and the ground state of $\mathrm{O}_{2}$ (R9), followed by the formation of endoperoxide/hydroperoxide derivatives (R10). ${ }^{84}$ These peroxide intermediates may also undergo further oxidation by $h_{V B}^{+}$or participate in reactions with $\mathrm{OH}$ radicals (R20) and be finally mineralized. In the case of solutions pre-bubbled with $\mathrm{O}_{2}$, the nanotube cavities are expected to be occupied with a sufficient number of $\mathrm{O}_{2}$ molecules, thereby making the endoperoxide/hydroperoxide intermediate pathway an important one. On the contrary, for solutions pre-bubbled with argon, direct electron transfer from $\mathrm{DBAN}^{*}(\mathrm{R} 6)$ and (DBAN) $)^{*}(\mathrm{R} 11)$ to Imo$\mathrm{CH}_{3}$ is likely to be the main pathway. However, even in Ar-bubbled solutions, the production of small concentrations of endoperoxide/hydroperoxide intermediates cannot be ruled out, as a minor concentration of $\mathrm{O}_{2}$ may be formed within the nanotube cavity by possible oxidation of water molecules, which are residually present inside the nanotube cavity as water vapor (R7). However, the concentration of formed $\mathrm{O}_{2}$, if any, is below the detection limit of the $\mu$-GC. In the case of the air-saturated system, both of these mechanistic pathways can exist. Moreover, the possibility of direct water photolysis using a short wavelength UV radiation could also generate reactive species (for example $\cdot \mathrm{OH}, \mathrm{O}_{2} \cdot-$ etc.) and hydrated electrons in the DBAN-Imo- $\mathrm{CH}_{3}$ aqueous solution. ${ }^{106-109}$ However, the yields of the species formed by direct water photolysis are expected to be much lower than those of the species produced by $\mathrm{Imo}-\mathrm{CH}_{3}$, and this route is therefore neglected here. The gaseous products such as $\mathrm{CO}, \mathrm{CH}_{4}$ and $\mathrm{H}_{2}$ are formed by further photo-reduction of $\mathrm{CO}_{2}$ and water 
on the external surface of the nanotubes (R21-23). Scheme 1 shows the major possible photodegradation pathways of DBAN encapsulated in $\mathrm{Imo}^{-} \mathrm{CH}_{3}$ nanotubes.

Table 1. Possible photo-redox reactions involving the photo-degradation of DBAN together with fuel production in an Imo-CH3 photo-reactor under UV illumination.

\begin{tabular}{|c|c|c|c|}
\hline \multicolumn{4}{|c|}{ Production of charge pairs } \\
\hline \multicolumn{3}{|c|}{$\operatorname{ImoCH}_{3}+h v \rightarrow e_{C B}^{-}+h_{V B}^{+}$} & R1 \\
\hline \multicolumn{2}{|l|}{ Photo-oxidation inside the cavity } & \multicolumn{2}{|c|}{ Photo-reduction on the external surface } \\
\hline$D B A N+h v \rightarrow D B A N^{*}$ & R2 & $O_{2}+e_{C B}^{-} \rightarrow O_{2}^{-}$ & $\mathrm{R} 12$ \\
\hline$D B A N^{*} \rightarrow D B A N$ & R3 & $\mathrm{O}_{2}^{-}+\mathrm{H}_{2} \mathrm{O}+e_{\mathrm{CB}}^{-} \rightarrow^{-\cdot} \mathrm{OOH}+{ }^{-} \cdot \mathrm{OH}$ & R13 \\
\hline$D B A N+D B A N^{*} \rightarrow\left(D B A N^{*}\right)_{2}^{\text {Excimer }}$ & $\mathrm{R} 4$ & ${ }^{-} \cdot \mathrm{OOH}+e_{\mathrm{CB}}^{-} \rightarrow \cdot \mathrm{O}_{\text {surf }}^{-}+{ }^{-} \cdot \mathrm{OH}$ & $\mathrm{R} 14$ \\
\hline$\rightarrow(D B A N)_{2}^{*}$ & & & \\
\hline$(D B A N)_{2}^{*} \rightarrow(D B A N)_{2}$ & R5 & $\mathrm{O}_{\text {surf }}^{-}+\mathrm{H}_{2} \mathrm{O}+e_{\mathrm{CB}}^{-} \rightarrow 2 \mathrm{OH}^{-}$ & R15 \\
\hline$D B A N\left(\right.$ or $\left.D B A N^{*}\right)+h_{V B}^{+} \rightarrow D B A N^{+} \rightarrow \rightarrow$ & R6 & ${ }^{-} \cdot \mathrm{OOH}+\mathrm{H}_{2} \mathrm{O} \rightarrow \mathrm{H}_{2} \mathrm{O}_{2}+{ }^{-} \cdot \mathrm{OH}$ & $\mathrm{R} 16$ \\
\hline$\rightarrow$ polymer & & & \\
\hline $2 \mathrm{H}_{2} \mathrm{O}+4 h_{V B}^{+} \rightarrow \mathrm{O}_{2}+4 H^{+}$ & $\mathrm{R} 7$ & $\mathrm{O}_{2}^{-}+H^{+} \rightarrow \cdot \mathrm{OOH}$ & $\mathrm{R} 17$ \\
\hline $\mathrm{H}_{2} \mathrm{O}+h_{V B}^{+} \rightarrow \mathrm{OH}+\mathrm{H}^{+}$ & $\mathrm{R} 8$ & $\cdot \mathrm{OOH}+\mathrm{OOH} \rightarrow \mathrm{H}_{2} \mathrm{O}_{2}+\mathrm{O}_{2}$ & R18 \\
\hline$D B A N^{*}+O_{2} \rightarrow D B A N+{ }^{1} O_{2}$ & R9 & $\mathrm{H}_{2} \mathrm{O}_{2}+e_{C B}^{-} \rightarrow \mathrm{OH}+\mathrm{OH}^{-}$ & $\mathrm{R} 19$ \\
\hline$D B A N+{ }^{1} O_{2} \rightarrow \rightarrow$ peroxide compound & $\mathrm{R} 10$ & & \\
\hline$(D B A N)_{2}^{*}+h_{V B}^{+} \rightarrow$ radical cation $\rightarrow \rightarrow$ & $\mathrm{R} 11$ & & \\
\hline$\rightarrow$ polymer & & & \\
\hline \multicolumn{4}{|c|}{ Mineralization inside the cavity } \\
\hline \multicolumn{3}{|c|}{$\mathrm{DBAN} /(\mathrm{DBAN})_{2} /$ polymers/intermediates $+\cdot \mathrm{OH} \rightarrow \rightarrow \rightarrow \mathrm{CO}_{2}+\mathrm{H}_{2} \mathrm{O}$} & R20 \\
\hline \multicolumn{4}{|c|}{ Fuel conversion on the external surface } \\
\hline $\mathrm{CO}_{2}+2 \mathrm{H}^{+}+2 e^{-} \rightarrow \mathrm{CO}+\mathrm{H}_{2} \mathrm{O}$ & & & R21 \\
\hline
\end{tabular}




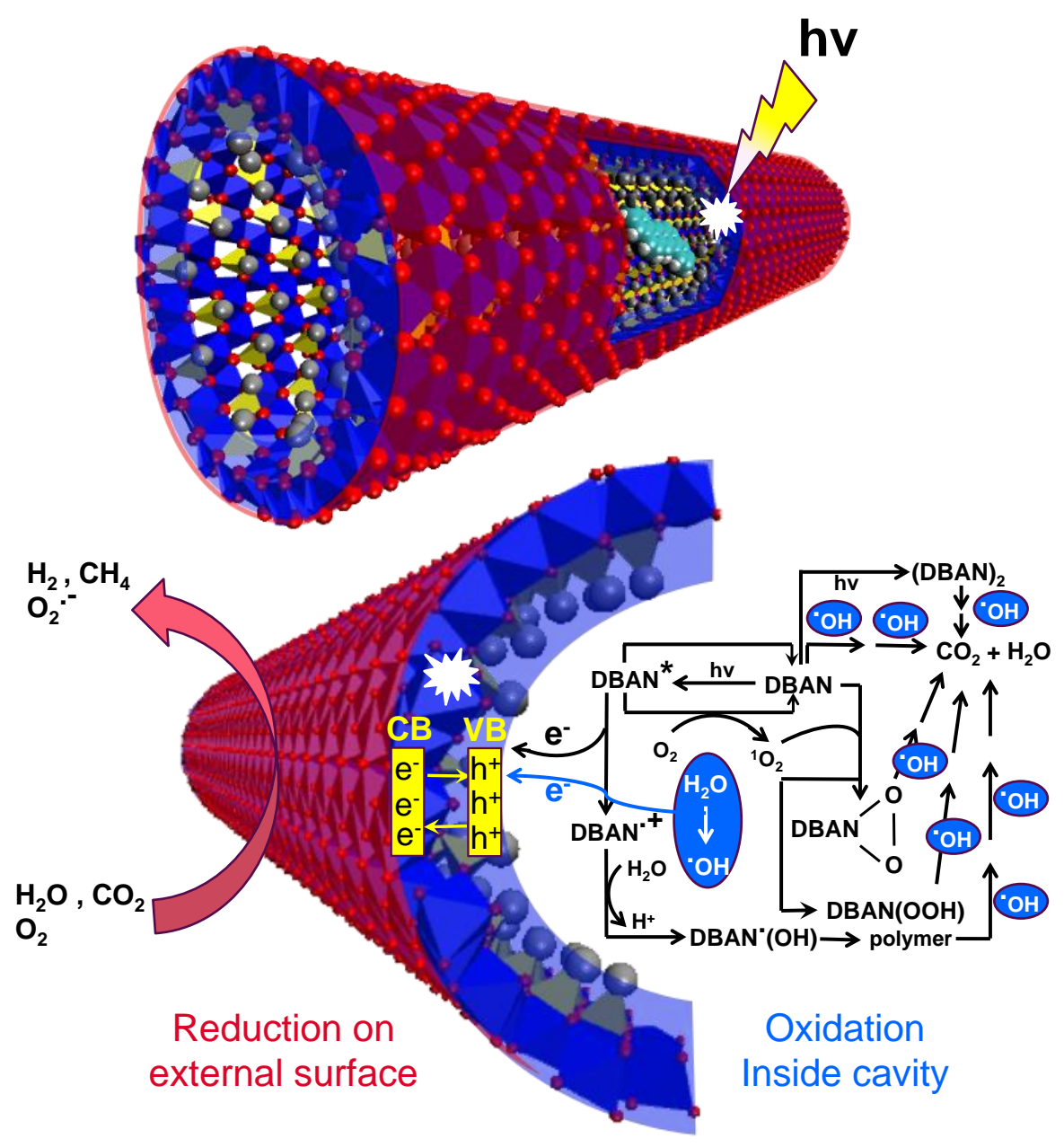

Scheme 1. Illustration of possible major photo-degradation pathways of DBAN encapsulated in Imo- $\mathrm{CH}_{3}$ nanotubes in aqueous medium.

\section{Conclusions}

The present work utilizes and demonstrates the predicted charge separation effect in inorganic aluminosilicate nanotubes (imogolite). It establishes the promising potential of Janus hybrid methyl imogolite nanotubes (Imo- $\left.\mathrm{CH}_{3}\right)$ as dual photocatalytic nanoreactors for simultaneous and efficient 
oxidation and reduction reactions inside and outside the nanotubes, respectively. Such a Janus nanoreactor is particularly suited for the treatment of water contaminated with hazardous organic pollutants. Imo- $\mathrm{CH}_{3}$ nanotubes, owing to their hydrophobic core, could efficiently encapsulate a phototoxic model pollutant, dibenzo(a,h)anthracene (DBAN), that was subsequently decomposed inside the Imo- $\mathrm{CH}_{3}$ nanocavity by photocatalytic oxidation under UV light. The encapsulated DBAN molecules underwent two parallel processes, photo-oxidation and photo-cycloaddition. Due to these parallel photochemical processes, the kinetics of photo-degradation of DBAN inside Imo$\mathrm{CH}_{3}$ is complex and depends on the initial DBAN concentration, but also on the concentration of dissolved dioxygen. UV degradation of DBAN or of the photo-dimers/oligomers inside $\mathrm{Imo}^{-\mathrm{CH}_{3}}$ nanotubes produces gases, such as $\mathrm{CO}_{2}, \mathrm{CO}$ and $\mathrm{CH}_{4}$ along with $\mathrm{H}_{2}$. The formation of $\mathrm{CH}_{4}$ and $\mathrm{H}_{2}$ evidences the role of the external surfaces of the nanotubes as photo-reduction centers.

By comparing the predicted energy band edge positions of $\mathrm{Imo}_{-} \mathrm{CH}_{3}$ nanotubes with popular existing photocatalytic systems, such as $\mathrm{TiO}_{2}, \mathrm{CdS}, \mathrm{CdSe}, \mathrm{ZnS}, \mathrm{SnO}_{2}, \mathrm{ZrO}_{2},{ }^{110} \mathrm{Imo}^{-\mathrm{CH}_{3}}$ is clearly a large band gap material. Its band edges are close in energy position to those of $\mathrm{ZrO}_{2}$, owing to a very similar band gap energy value. ${ }^{111,112}$ However, $\mathrm{Imo}^{-\mathrm{CH}_{3}}$ possesses numerous advantages due to its (i) environmentally benign chemical composition, (ii) low cost of synthesis, (iii) hollow Janus nanostructure with an hydrophobic internal cavity favoring the encapsulation of organic species and an hydrophilic external surface favoring the dispersion of the nanotubes in water, (iv) favored curvature-induced charge separation across the polarized wall, (v) tunable surface property etc. Further studies on the band gap reduction of doped $\mathrm{Imo}-\mathrm{CH}_{3}$, better charge separation by using metal nanoparticle reactive centers on the external wall or suitable photoactive antenna molecules could enable such nano-photo-reactors to work efficiently under the solar spectrum. The present discovery of hybrid Imo- $\mathrm{CH}_{3}$ as a dual photocatalytic nano-reactor could strengthen imogolitebased photocatalytic research and accelerate many technological breakthroughs for solar photocatalysis by combining two popular but otherwise disconnected photochemical reactions (i.e. pollutant mineralization and production of fuels such as $\mathrm{H}_{2}, \mathrm{CH}_{4}$ ).

\section{Electronic Supplementary Information}

TEM images, SAXS data, FT-IR characterization, additional UV-Vis absorption and fluorescence emission data, fitting of kinetic curves, stability of nanoreactor. 


\section{Conflict of Interest}

The authors declare no competing financial interest.

\section{Acknowledgements}

An Ile de France DIM RESPORE 2019-20 and CEA Bottom Up 2020-21 grant funded this study by the project GRANITE and PHOTOTUBE, respectively. S.P. gratefully acknowledges the DIM

RESPORE and the CEA for the post-doctoral fellowship. Dr. Frederic Gobeaux is sincerely acknowledged for the transmission electron microscopy images. Authors thank Dr. Mark Levenstein for reading the manuscript and making insightful comments. The project proposal GRANITE, submitted jointly by S.P. and A.T., was awarded "Marie Sklodowska-Curie Actions Seal of Excellence" from the Marie Skłodowska-Curie actions call H2020-MSCA-IF-2018.

\section{Notes and References}

1. A. Fujishima, K. Honda, Electrochemical Photolysis of Water at a Semiconductor Electrode, Nature 1972, 238, 37.

2. X. Chen, S.S. Mao, Titanium Dioxide Nanomaterials: Synthesis, Properties, Modifications, and Applications, Chem. Rev. 2007, 107, 2891.

3. A. Fujishima, T.N. Rao, D.A. Tryk, Titanium dioxide photocatalysis, J. Photochem. Photobiol. C: Photochemistry Reviews 2000, 1, 1.

4. D. Chatterjee, S. Dasgupta, Visible light induced photocatalytic degradation of organic pollutants, J. Photochem. Photobiol. C: Photochemistry Reviews 2005, 6, 186.

5. X. Chen, L. Liu, P.Y. Yu, S.S. Mao, Increasing solar absorption for photocatalysis with black hydrogenated titanium dioxide nanocrystals, Science 2011, 331, 746.

6. X. Zong, H. Yan, G. Wu, G. Ma, F. Wen, L. Wang, C. Li, Enhancement of Photocatalytic $\mathrm{H}_{2}$ Evolution on CdS by Loading $\mathrm{MoS}_{2}$ as Cocatalyst under Visible Light Irradiation, $J$. Am. Chem. Soc. 2008, 130, 7176.

7. W. Xu, S. Zhu, Y. Liang, Z. Li, Z. Cui, X. Yang, A. Inoue, Nanoporous CuS with excellent photocatalytic property, Scientific Reports 2015, 5, 18125.

8. T.W. Kim, K.-S. Choi, Nanoporous $\mathrm{BiVO}_{4}$ Photoanodes with Dual-Layer Oxygen Evolution Catalysts for Solar Water Splitting, Science 2014, 343, 990.

9. D. Chen, J. Ye, Hierarchical $\mathrm{WO}_{3}$ Hollow Shells: Dendrite, Sphere, Dumbbell, and Their Photocatalytic Properties, Adv. Funct. Mater. 2008, 18, 1922.

10. V. Vaiano, M. Matarangolo, J.J. Murcia, H. Rojas, J.A. Navíoc, M.C. Hidalgo, Enhanced photocatalytic removal of phenol from aqueous solutions using $\mathrm{ZnO}$ modified with $\mathrm{Ag}$, Applied Catalysis B: Environmental 2018, 225, 197.

11. H.S. Jung, Y.J. Hong, Y. Li, J. Cho, Y.-J. Kim, G.-C. Yi, Photocatalysis Using GaN Nanowires, ACS Nano 2008, 2, 637.

12. K. Maeda, K. Teramura, D. Lu, T. Takata, N. Saito, Y. Inoue, K. Domen, Photocatalyst releasing hydrogen from water, Nature 2006, 440, 295. 
13. G. Zhang, G. Liu, L. Wang, J.T.S. Irvine, Inorganic perovskite photocatalysts for solar energy utilization, Chem. Soc. Rev., 2016, 45, 5951.

14. X. Wang, K. Maeda, A. Thomas, K. Takanabe, G. Xin, J.M. Carlsson, K. Domen, M. Antonietti, A metal-free polymeric photocatalyst for hydrogen production from water under visible light, Nat. Mater. 2009, 8, 76.

15. A. Khlyustova, N. Sirotkin, T. Kusova, A. Kraev, V. Titov, A. Agafonov, Doped $\mathrm{TiO}_{2}$ : the effect of doping elements on photocatalytic activity, Mater. Adv., 2020, 1, 1193.

16. J.S. Schubert, J. Popovic, G.M. Haselmann, S.P. Nandan, J. Wang, A. Giesriegl, A.S. Cherevan, D. Eder, Immobilization of $\mathrm{Co}, \mathrm{Mn}, \mathrm{Ni}$ and $\mathrm{Fe}$ oxide cocatalysts on $\mathrm{TiO}_{2}$ for photocatalytic water splitting reactions, J. Mater. Chem. A 2019, 7, 18568.

17. S. Chen, T. Takata, K. Domen, Particulate photocatalysts for overall water splitting, Nat. Rev. Mater. 2017, 2, 17050.

18. M. Xiao, Z. Wang, M. Lyu, B. Luo, S. Wang, G. Liu, H-M. Cheng, L. Wang, Hollow Nanostructures for Photocatalysis: Advantages and Challenges, Adv. Mater. 2019, 31, 1801369.

19. F. Chen, H. Huang, L. Guo, Y. Zhang, T. Ma, The Role of Polarization in Photocatalysis, Angew. Chem. Int. Ed. 2019, 58, 10061.

20. E. Poli, J.D. Elliott, L.E. Ratcliff, L. Andrinopoulos, J. Dziedzic, N.D.M. Hine, A.A. Mostofi, C.-K. Skylaris, P.D. Haynes, G. Teobaldi, The potential of imogolite nanotubes as (co-) photocatalysts: a linear-scaling density functional theory study, J. Phys.: Condens. Matter 2016, 28, 074003.

21. J.D. Elliott, E. Poli, I. Scivetti, L.E. Ratcliff, L. Andrinopoulos, J. Dziedzic, N.D.M. Hine, A.A. Mostofi, C-K. Skylaris, P.D. Haynes, G. Teobaldi. Chemically Selective Alternatives to Photoferroelectrics for Polarization-Enhanced Photocatalysis: The Untapped Potential of Hybrid Inorganic Nanotubes, Adv. Sci. 2017, 4, 1600153.

22. M. Pignie, V. Shcherbakov, T. Charpentier, M. Moskura, C. Carteret, S.A. Denisov, M. Mostafavi, A. Thill, S. Le Caër, Confined Water Radiolysis in Aluminosilicate Nanotubes: The Importance of Charge Separation Effects, Nanoscale 2021, 13, 3092.

23. N. Yoshinaga, S. Aomine, Imogolite in some ando soils. Soil Sci. Plant Nutr. 1962, 8.

24. E. Paineau, M-E.M. Krapf, M-S. Amara, N.V. Matskova, I. Dozov, S. Rouzière, A. Thill, P. Launois, P. Davidson, A liquid-crystalline hexagonal columnar phase in highly-dilute suspensions of imogolite nanotubes, Nat. Comm. 2016, 7, 10271.

25. M-S. Amara, E. Paineau, M. Bacia-Verloop, M-E.M. Krapf, P. Davidson, L. Belloni, C. Levard, J. Rose, P. Launois, A. Thill, A. Single-step formation of micron long $(\mathrm{OH})_{3} \mathrm{Al}_{2} \mathrm{O}_{3} \mathrm{Ge}(\mathrm{OH})$ imogolite-like nanotubes, Chem. Commun. 2013, 49, 11284.

26. M. Zhao, Y. Xia, L. Mei, Energetic Minimum Structures of Imogolite Nanotubes: A FirstPrinciples Prediction, J. Phys. Chem. C 2009, 113, 14834.

27. E. Poli, J.D. Elliott, S.K. Chulkov, M.B. Watkins, G. Teobaldi, The Role of CationVacancies for the Electronic and Optical Properties of Aluminosilicate Imogolite Nanotubes: A Non-local, Linear-Response TDDFT, Front. Chem. 2019, 7, 210.

28. J.S. Jang, U.A. Joshi, J.S. Lee, Solvothermal Synthesis of CdS Nanowires for Photocatalytic Hydrogen and Electricity Production, J. Phys. Chem. C 2007, 111, 13280.

29. G. Kenanakis, N. Katsarakis, Light-induced photocatalytic degradation of stearic acid by c-axis oriented ZnO nanowires, Appl. Catal. A: Gen. 2010, 378, 227.

30. C. Chen, Z. Li, H. Lin, G. Wang, J. Liao, M. Li, S. Lv, W. Li, Enhanced visible light photocatalytic performance of $\mathrm{ZnO}$ nanowires integrated with $\mathrm{CdS}$ and $\mathrm{Ag}_{2} \mathrm{~S}$, Dalton Trans. 2016, 45, 3750. 
31. D. Hong, W. Zang, X. Guo, Y. Fu, H. He, J. Sun, L. Xing, B. Liu, X. Xue, High Piezophotocatalytic Efficiency of $\mathrm{CuS} / \mathrm{ZnO}$ Nanowires Using Both Solar and Mechanical Energy for Degrading Organic Dye, ACS Appl. Mater. Interfaces 2016, 8, 21302.

32. Q. Liu, Y. Zhou, J. Kou, X. Chen, Z. Tian, J. Gao, S. Yan, Z. Zou, High-Yield Synthesis of Ultralong and Ultrathin $\mathrm{Zn}_{2} \mathrm{GeO}_{4}$ Nanoribbons toward Improved Photocatalytic Reduction of $\mathrm{CO}_{2}$ into Renewable Hydrocarbon Fuel, J. Am. Chem. Soc. 2010, 132, 14385.

33. L. Soto-Vázqueza, M. Cotto, J. Ducongé, C. Morant, F. Márquez, Synthesis and photocatalytic activity of $\mathrm{TiO}_{2}$ nanowires in the degradation of p-aminobenzoic acid: A comparative study with a commercial catalyst, J. Environmental Management 2016, 167, 23.

34. G.I. Yucelen, D-Y. Kang, R.C. Guerrero-Ferreira, E.R. Wright, H.W. Beckham, S. Nair, Shaping single-walled metal oxide nanotubes from precursors of controlled curvature, Nano Lett. 2012, 12, 827.

35. K. Yamamoto, H. Otsuka, S.I. Wada, D. Sohn, A. Takahara, Preparation and properties of poly(methyl methacrylate)/imogolite hybrid via surface modification using phosphoric acid ester, Polymer 2005, 46, 12386.

36. B. Thomas, T. Coradin, G. Laurent, R. Valentin, Z. Mouloungui, F. Babonneau, N. Baccile, Biosurfactant-mediated one-step synthesis of hydrophobic functional imogolite nanotubes, RSC Advances 2012, 2, 426.

37. B.H. Bac, Y. Song, M.H. Kim, Y.-B. Lee, I.M. Kang, Surface-modified aluminogermanate nanotube by OPA: synthesis and characterization, Inorg. Chem. Commun. 2009, 12, 1045.

38. W. Ma, H. Otsuka, A. Takahara, Preparation and properties of PVC/PMMA-g imogolite nanohybrid via surface-initiated radical polymerization, Polymer 2011, 52, 5543.

39. B. Bonelli, M. Armandia, E. Garrone, Surface properties of alumino-silicate single-walled nanotubes of the imogolite type, Phys. Chem. Chem. Phys., 2013, 15, 13381.

40. E. Shafia, S. Esposito, M. Manzoli, M. Chiesa, P. Tiberto, G. Barrera, G. Menard, P. Allia, F.S. Freyria, E. Garrone, B. Bonelli, Al/Fe isomorphic substitution versus $\mathrm{Fe}_{2} \mathrm{O}_{3}$ clusters formation in Fe-doped aluminosilicate nanotubes (imogolite), J. Nanopart. Res. 2015, 17, 336.

41. E. Shafia, S. Esposito, M. Armandi, M. Manzoli, E. Garrone, B. Bonelli, Isomorphic substitution of aluminium by iron into single-walled alumino-silicate nanotubes: A physico-chemical insight into the structural and adsorption properties of Fe-doped imogolite, Microporous and Mesoporous Materials 2016, 224, 229.

42. E. Shafia, S. Esposito, M. Armandi, E. Bahadori, E. Garrone, B. Bonelli, Reactivity of bare and Fe-doped alumino-silicate nanotubes (imogolite) with $\mathrm{H}_{2} \mathrm{O}_{2}$ and the azo-dye Acid Orange 7, Catalysis Today 2016, 277, 89.

43. E. Bahadori, V. Vaiano, S. Esposito, M. Armandi, D. Sannino, B. Bonelli, Photo-activated degradation of tartrazine by $\mathrm{H}_{2} \mathrm{O}_{2}$ as catalyzed by both bare and $\mathrm{Fe}$-doped methyl-imogolite nanotubes, Catalysis Today 2018, 304, 199.

44. I. Bottero, B. Bonelli, S.E. Ashbrook, P.A. Wright, W. Zhou, M. Tagliabue, M. Armandi, E. Garrone, Synthesis and characterization of hybrid organic/inorganic nanotubes of the imogolite type and their behaviour towards methane adsorption, Phys. Chem. Chem. Phys. 2011,13, 744.

45. M. Boyer, E. Paineau, M. Bacia-Verloop, A. Thill, Aqueous dispersion state of amphiphilic hybrid aluminosilicate nanotubes, Applied Clay Science 2014, 96, 45. 
46. P. Picot, O. Taché, F. Malloggi, T. Coradinb, A. Thill, Behaviour of hybrid inside/out Janus nanotubes at an oil/water interface. A route to self-assembled nanofluidics?, Faraday Discuss. 2016, 191, 391.

47. P. Picot, Y. Liao, E. Barruet, F. Gobeaux, T. Coradin, A. Thill, Exploring Hybrid Imogolite Nanotube Formation via Si/Al Stoichiometry Control, Langmuir 2018, 34, 13225.

48. M.S. Amara, E. Paineau, S. Rouzière, B. Guiose, M-E.M. Krapf, O. Taché, P. Launois, A. Thill, Hybrid, Tunable-Diameter, Metal Oxide Nanotubes for Trapping of Organic Molecules, Chem. Mater. 2015, 27, 1488.

49. P. Picot, F. Gobeaux, T. Coradin, A. Thill, Dual internal functionalization of imogolite nanotubes as evidenced by optical properties of Nile red, Appl. Clay Sci. 2019, 178, 105133.

50. R. Nasi, F. Sannino, P. Picot, A. Thill, O. Oliviero, S. Esposito, M. Armandi, B. Bonelli, Hybrid organic-inorganic nanotubes effectively adsorb some organic pollutants in aqueous phase, Appl. Clay Sci. 2020, 186, 105449.

51. G. Monet, M.S. Amara, S. Rouzière, E. Paineau, Z. Chai, J.D. Elliott, E. Poli, L-M. Liu, G. Teobaldi, P. Launois, Structural resolution of inorganic nanotubes with complex stoichiometry, Nature Communications 2018, 9, 2033.

52. E. Kriek, M. Rojas, K. Alexandrov, H. Bartsch, Polycyclic aromatic hydrocarbon-DNA adducts in humans: relevance as biomarkers for exposure and cancer risk. Mutat. Res. 1998, 400, 215.

53. C. Bosetti, P. Boffetta, C. La Vecchia, Occupational exposures to polycyclic aromatic hydrocarbons, and respiratory and urinary tract cancers: a quantitative review to 2005 . Annals Oncol. 2007, 18, 431.

54. I. Burstyn, H. Kromhout, C. Johansen, S. Langard, T. Kauppinen, J. Shaham, G. Ferro, P. Boffetta, Bladder cancer incidence and exposure to polycyclic aromatic hydrocarbons among asphalt pavers. Occup. Environ. Med. 2007, 64, 520.

55. L.K. Siddens, A. Larkin, S.K. Krueger, C.A. Bradfield, K.M. Waters, S.C. Tilton, C.B. Pereira, C.V. Löhr, V.M. Arlt, D.H. Phillips, D.E. Williams, W.M. Baird, Polycyclic aromatic hydrocarbons as skin carcinogens: comparison of benzo[a]pyrene, dibenzo[def,p]chrysene and three environmental mixtures in the FVB/N mouse. Toxicol. Appl. Pharmacol. 2012, 264, 377.

56. A.C. Hatch, G.A. Burton Jr., Photo-induced toxicity of PAHs to Hyalella azteca and Chironomus tentans: effects of mixtures and behavior, Environ. Pollut. 1999, 106, 157.

57. A. Mallakin, D.G. Dixxon, B.M. Greenberg, Pathway of anthracene modification under simulated solar radiation, Chemosphere 2000, 40, 1435.

58. G.T. Ankley, R.J. Erickson, G.L. Phipps, V.R. Mattson, P.A. Kosian, B.R. Sheedy, J.S. Cox, Effects of light intensity on the phototoxicity of fluoranthene to a benthic macroinvertebrate, Environ. Sci. Technol. 1995, 29, 2828.

59. W.M. Baird, L.A. Hooven, B. Mahadevan, Carcinogenic polycyclic aromatic hydrocarbonDNA adducts and mechanism of action, Environ. Mol. Mutagen. 2005, 45, 106.

60. F. Alves de Lima Ribeiro, M. Miguel Castro Ferreira, QSAR model of the phototoxicity of polycyclic aromatic hydrocarbons, J. Mol. Struct. (Theochem) 2005, 719, 191.

61. S.E. Manahan, Environmental Chemistry, 6th ed., Lewis Publishers, Boca Ranton, 1994. chapter 7.

62. A.F. Lehner, J. Horn, J.W. Flesher, Benzylic carbonium ions as ultimate carcinogens of polynuclear aromatic hydrocarbons, J. Mol. Struct. (Theochem) 1996, 366, 203.

63. C. Baird, Environmental Chemistry, 2nd ed., Freeman, W.H. and Company, USA, 1995. chapter 14 . 
64. R. Abou-Naccoul, I. Mokbel, G. Bassil, J. Saab, K. Stephan, J. Jose, Aqueous solubility (in the range between 298.15 and $338.15 \mathrm{~K}$ ), vapor pressures (in the range between 105 and 80 $\mathrm{Pa}$ ) and Henry's law constant of 1,2,3,4-dibenzanthracene and 1,2,5,6-dibenzanthracene, Chemosphere 2014, 95, 41.

65. P. Ruelle, M. Buchmann, H. Nam-Tran, U.W. Kesselring, Enhancement of the solubilities of polycyclic aromatic hydrocarbons by weak hydrogen bonds with water, Journal of Computer-Aided Molecular Design 1992, 6, 431.

66. J.R. Boltona, M.I. Stefanb, P-S. Shawc, K.R. Lykkec, Determination of the quantum yields of the potassium ferrioxalate and potassium iodide-iodate actinometers and a method for the calibration of radiometer detectors, J. Photochem. Photobiol. A: Chem. 2011, 222, 166.

67. S. Le Caër, M. Pignié, Q. Berrod, V. Grzimek, M. Russina, C. Carteret, A. Thill, J. Zanotti, J. Teixeira, Dynamics in hydrated inorganic nanotubes studied by neutron scattering: towards nanoreactors in water, Nanoscale Adv., 2021, 3, 789.

68. D.V. Kazachkin, Y. Nishimura, H.A. Witek, Stephan Irle, E. Borguet, Dramatic Reduction of IR Vibrational Cross Sections of Molecules Encapsulated in Carbon Nanotubes, J. Am. Chem. Soc. 2011, 133, 8191.

69. J. Tropp, M.H. Ihde, A.K. Williams, N.J. White, N. Eedugurala, N.C. Bell, J.D. Azoulay, M. Bonizzoni, A sensor array for the discrimination of polycyclic aromatic hydrocarbons using conjugated polymers and the inner filter effect, Chem. Sci., 2019, 10, 10247.

70. R.N. Jones, The Ultraviolet Absorption Spectra of Anthracene Derivatives, Chem. Rev. 1947, 41, 353.

71. K. Carter-Fenk, J.M. Herbert, Reinterpreting p-stacking, Phys. Chem. Chem. Phys. 2020, 22, 24870.

72. S.E. Fioressi, R.C. Binning Jr., D.E. Bacelo, Effects of cluster formation on spectra of benzo[a]pyrene and benzo[e]pyrene, Chem. Phys. Lett., 2008, 454, 269.

73. K-M. Lehto, E. Vuorimaa, H. Lemmetyinen, Photolysis of polycyclic aromatic hydrocarbons (PAHs) in dilute aqueous solutions detected by fluorescence, J. Photochem. Photobiol. A: Chem. 2000, 136, 53.

74. Y. Liao, P. Picot, M. Lainé, J-B. Brubach, P. Roy, A. Thill, S. Le Caër, Tuning the properties of confined water in standard and hybrid nanotubes: An infrared spectroscopic study, Nano Research 2018, 11(9), 4759.

75. G.K.-C. Low, G.B. Batley, C.I. Brockbank, Solvent induced photodegradation as a source of error in the analysis of polycyclic aromatic hydrocarbons, J. Chromatogr. 1987, 392, 199.

76. H-D. Becker, Unimolecular Photochemistry of Anthracenes, Chem. Rev. 1993, 93, 145.

77. H. Bouas-Laurent, A. Castellan, J-P. Desvergne, R. Lapouyade, Photodimerization of anthracenes in fluid solution: structural aspects, Chem. Soc. Rev. 2000, 29, 43.

78. H. Bouas-Laurent, A. Castellan, J.-P. Desvergne, R. Lapouyade, Photodimerization of anthracenes in fluid solutions: (part 2) mechanistic aspects of the photocycloaddition and of the photochemical and thermal cleavage, Chem. Soc. Rev. 2001, 30, 248.

79. S. Singh, C. Sandorf, Infrared spectra of photodimers of anthracene, benzo[a]anthracene, and tetracene, Canadian J. Chem. 1969, 47, 257.

80. D. Han, H. Lu, W. Li, Y. Li, S. Feng, Light- and heat-triggered reversible luminescent materials based on polysiloxanes with anthracene groups, RSC Adv. 2017, 7, 56489.

81. V. Librando, G. Bracchitta, G.D. Guidi, Z. Minniti, G. Perrini, A. Catalfo, Photodegradation of Anthracene and Benzo[a]anthracene in Polar and Apolar Media: New Pathways of Photodegradation, Polycyclic Aromatic Compounds 2014, 34, 263. 
82. J. Tanabe, D. Taura, N. Ousaka, E. Yashima, Remarkable acceleration of template-directed photodimerisation of 9-phenylethynylanthracene derivatives assisted by complementary salt bridge formation, Org. Biomol. Chem. 2016, 14, 10822.

83. M. Saito, I. Sakon, C. Kaito, Y. Kimura, Formation of polycyclic aromatic hydrocarbon grains using anthracene and their stability under UV irradiation, Earth Planets Space 2010, 62, 81 .

84. J.S. Miller, D. Olejnik, Photolysis of polycyclic aromatic hydrocarbons in water, Wat. Res. 2001, 35, 233.

85. Y.M. Lopatkin, P.A. Kondratenko, I.P. Zharkov, Kinetics of the photodimerisation of anthracene in an inhomogeneous polymer matrix, Theoretical and Experimental Chemistry 2001, 37, 84.

86. T.F. Kahan, D.J. Donaldson, Photolysis of Polycyclic Aromatic Hydrocarbons on Water and Ice Surfaces, J. Phys. Chem. A 2007, 111, 1277.

87. R. Majidi, A. Karami, Band gap modulation of graphene and graphyne via tetracyanoethylene adsorption, Studia UBB Chemia, 2016, LXI, 1, 177.

88. B. Akdim, S.N. Kim, R.R. Naik, B. Maruyama, M.J. Pender, R. Pachter, Understanding effects of molecular adsorption at a single-wall boron nitride nanotube interface from density functional theory calculations, Nanotechnology 2009, 20, 355705.

89. P. Makuła, M. Pacia, W. Macyk, How To Correctly Determine the Band Gap Energy of Modified Semiconductor Photocatalysts Based on UV-Vis Spectra, J. Phys. Chem. Lett. 2018, 9, 6814.

90. W. Chu, C.T. Jafvert, Photodechlorination of Polychlorobenzene Congeners in Surfactant Micelle Solutions, Environ. Sci. Technol. 1994, 28, 2415.

91. S.M. Tsui, W. Chu, Quantum yield study of the photodegradation of hydrophobic dyes in the presence of acetone sensitizer, Chemosphere 2001, 44, 17.

92. R. Singh, S. Dutta, A review on $\mathrm{H}_{2}$ production through photocatalytic reactions using TiO2/TiO2-assisted catalysts, Fuel 2018, 220, 607.

93. N. Li, B. Wang, Y. Si, F. Xue, J. Zhou, Y. Lu, M. Liu, Toward High-Value Hydrocarbon Generation by Photocatalytic Reduction of CO2 in Water Vapor, ACS Catal. 2019, 9, 5590.

94. Z. Long, Q. Li, T. Wei, G. Zhanga, Z. Ren, Historical development and prospects of photocatalysts for pollutant removal in water, Journal of Hazardous Materials 2020, 395, 122599.

95. K.C. Christoforidis, P. Fornasiero, Photocatalytic Hydrogen Production: A Rift into the Future Energy Supply, ChemCatChem 2017, 9, 1523.

96. Y. Matsumoto, Energy Positions of Oxide Semiconductors and Photocatalysis with Iron Complex Oxides, Journal of Solid State Chemistry 1996, 126, 227.

97. K. Li, B. Peng, T. Peng, Recent Advances in Heterogeneous Photocatalytic CO2 Conversion to Solar Fuels, ACS Catal. 2016, 6, 7485.

98. S. Siahrostami, G-L. Li, V. Viswanathan, J.K. Nørskov, One- or Two-Electron Water Oxidation, Hydroxyl Radical, or $\mathrm{H}_{2} \mathrm{O}_{2}$ Evolution, J. Phys. Chem. Lett. 2017, 8, 1157.

99. P. Singh, K. Sharma, V. Hasija, V. Sharma, S. Sharma, P. Raizada, M. Singh, A.K. Saini, A. Hosseini-Bandegharaei, V.K. Thakur, Systematic review on applicability of magnetic iron oxideseintegrated photocatalysts for degradation of organic pollutants in water, Materials Today Chemistry 2019, 14, 100186.

100. Polycyclic Arenes and Heteroarenes: Synthesis, properties and applications, Editor: Miao, Q. 2016, Wiley VCH Verlag GmbH \& Co. 
101. G. Reginato, L. Zani, M. Calamante, A. Mordini, A. Dessì, Dye-Sensitized Heterogeneous Photocatalysts for Green Redox Reactions, Eur. J. Inorg. Chem. 2020, 899917.

102. J.G. Radich, A.L. Krenselewski, J. Zhu, P.V. Kamat, Is Graphene a Stable Platform for Photocatalysis? Mineralization of Reduced Graphene Oxide With UV-Irradiated $\mathrm{TiO}_{2}$ Nanoparticles, Chem. Mater. 2014, 26, 4662.

103. L. Rojas, A. Peraza, F. Ruette, Aging Oxidation Reactions on Atmospheric Black Carbon by OH Radicals. A Theoretical Modeling Study, J. Phys. Chem. A 2015, 119, 13038 .

104. S. Wen, J. Zhao, G. Sheng, J. Fu, P. Peng, Photocatalytic reactions of pyrene at $\mathrm{TiO}_{2} /$ water interfaces, Chemosphere 2003, 50, 111.

105. M. Setvin, U. Aschauer, J. Hulva, T. Simschitz, B. Daniel, M. Schmid, A. Selloni, U. Diebold, Following the Reduction of Oxygen on $\mathrm{TiO}_{2}$ Anatase (101) Step by Step, $J$. Am. Chem. Soc. 2016, 138, 9565.

106. A. Chauhan, M. Rastogi, P. Scheier, C. Bowen, R.V. Kumar, R. Vaish, Janus nanostructures for heterogeneous photocatalysis, Appl. Phys. Rev. 2018, 5, 041111.

107. K. Azrague, E. Bonnefille, V. Pradines, V. Pimienta, E. Oliveros, M.-T. Maurette, F. Benoit-Marqui, Hydrogen peroxide evolution during V-UV photolysis of water, Photochem. Photobiol. Sci., 2005, 4, 406.

108. A. Kumar, M. Kolaski, H.M. Lee, K.S. Kim, Photoexcitation and Photoionization Dynamics of Water Photolysis, J. Phys. Chem. A 2008, 112, 5502.

109. K. Tomanova, M. Precek, V. Mucka, L. Vysin, L. Juhabc, V. Cuba, At the crossroad of photochemistry and radiation chemistry: formation of hydroxyl radicals in diluted aqueous solutions exposed to ultraviolet radiation, Phys. Chem. Chem. Phys. 2017, 19, 29402.

110. Y. Xu, M.A.A. Schoonen, The absolute energy positions of conduction and valence bands of selected semiconducting minerals, Am. Mineralogist, 2000, 85, 543.

111. C. Karunakaran, S. Senthilvelan, Photocatalysis with $\mathrm{ZrO}_{2}$ : oxidation of aniline, Journal of Molecular Catalysis A: Chemical 2005, 233, 1.

112. S. G. Botta, J. A. Navio, M. C. Hidalgo, G. M. Restrepo and M. Litter, Photocatalytic properties of $\mathrm{ZrO}_{2}$ and $\mathrm{Fe} / \mathrm{ZrO}_{2}$ semiconductors prepared by a sol-gel technique, J. Photochem. Photobiol. A: Chem., 1999, 129, 89. 